J. Korean Math. Soc. 46 (2009), No. 4, pp. 785-812

DOI 10.4134/JKMS.2009.46.4.785

\title{
WEAK METRIC AND WEAK COMETRIC SCHEMES
}

\author{
Dae San Kim and Gil Chun Kim
}

\begin{abstract}
The notion of weak metric and weak cometric schemes are introduced as a generalization of metric and cometric schemes. They are given as the wreath product of a finite number of symmetric association schemes satisfying certain equivalent conditions which are analogous to the ones for metric or cometric schemes. We characterize those schemes and determine some of their parameters.
\end{abstract}

\section{Introduction}

Let $\mathbb{F}_{q}$ be the finite field with $q$ elements, and let $\mathbb{P}=([n], \leq)$ be a poset on the underlying set $[n]=\{1,2, \ldots, n\}$ of coordinate positions of vectors in $\mathbb{F}_{q}^{n}$. Then the $\mathbb{P}$-weight $w_{\mathbb{P}}$ is the function on $\mathbb{F}_{q}^{n}$ which is given by

$$
w_{\mathbb{P}}(x)=\mid\{i \in[n] \mid i \leq j \text { for some } j \in \operatorname{Supp}(x)\} \mid .
$$

Here $\operatorname{Supp}(x)=\left\{j \in[n] \mid x_{j} \neq 0\right\}$ for $x=\left(x_{1}, \ldots, x_{n}\right) \in \mathbb{F}_{q}^{n}$. Now, $d_{\mathbb{P}}(x, y)=$ $w_{\mathbb{P}}(x-y)$ is a metric, called $\mathbb{P}$-metric. If $\mathbb{P}$ is an antichain, $\mathbb{P}$-weight and $\mathbb{P}$-metric reduce respectively to Hamming weight and Hamming metric. The notion of $\mathbb{P}$-codes, namely subsets $\mathcal{C} \subseteq \mathbb{F}_{q}^{n}$ equipped with $w_{\mathbb{P}}$, were introduced in [4] by Brualdi et. al.

For each linear code $\mathcal{C} \subseteq \mathbb{F}_{q}^{n}$, the $\mathbb{P}$-weight distribution of $\mathcal{C}$ is $\left\{A_{\mathbb{P}, i}(\mathcal{C})\right\}_{i=0}^{n}$, where

$$
A_{\mathbb{P}, i}(\mathcal{C})=\left|\left\{x \in \mathcal{C} \mid w_{\mathbb{P}}(x)=i\right\}\right| .
$$

We will denote by $\operatorname{Aut}\left(\mathbb{F}_{q}^{n}, w_{\mathbb{P}}\right)$ the group of all linear automorphisms $\tau: \mathbb{F}_{q}^{n} \rightarrow$ $\mathbb{F}_{q}^{n}$ satisfying $w_{\mathbb{P}}(\tau x)=w_{\mathbb{P}}(x)$ for all $x \in \mathbb{F}_{q}^{n}$. Let $\mathbb{P}_{0}=n_{1} \mathbf{1} \oplus \cdots \oplus n_{t} \mathbf{1}$ be the poset $\left(\mathbb{P}_{0}\right.$ is called a weak order poset) given as the ordinal sum of the antichains $n_{i} \mathbf{1}$ on the set $\left\{n_{1}+\cdots+n_{i-1}+1, \ldots, n_{1}+\cdots+n_{i-1}+n_{i}\right\}$ for $i=1, \ldots, t$, i.e., the underlying set is $[n]\left(n=n_{1}+\cdots+n_{t}\right)$ and the order relation is given by:

$$
i<j \Leftrightarrow i \in n_{l} \mathbf{1}, j \in n_{m} \mathbf{1} \text { for some } l<m .
$$

Received October 19, 2007.

2000 Mathematics Subject Classification. 05E30, 94B60.

Key words and phrases. weak metric scheme, weak cometric scheme.

This work was supported by grant No.R01-2006-000-11176-0 from the Basic Research Program of the Korea Science and Engineering Foundation. 
Then we have the following fundamental result.

Theorem A. The following are equivalent.

(1) $\mathbb{P}$ is a weak order poset on $[n]$.

(2) $\left(\mathbb{F}_{q}^{n},\left\{R_{i}\right\}_{i=0}^{n}\right)$, with $(x, y) \in R_{i} \Leftrightarrow d_{\mathbb{P}}(x, y)=i(0 \leq i \leq n)$ is a symmetric translation association scheme.

(3) The $\mathbb{P}$-weight distribution $\left\{A_{\mathbb{P}, i}(\mathcal{C})\right\}_{i=0}^{n}$ of $\mathcal{C}$ uniquely determines $\breve{\mathbb{P}}$ weight distribution $\left\{A_{\breve{\mathbb{P}}, i}\left(\mathcal{C}^{\perp}\right)\right\}$ of $\mathcal{C}^{\perp}$ for any linear code $\mathcal{C} \subseteq \mathbb{F}_{q}^{n}$.

(4) The group $\operatorname{Aut}\left(\mathbb{F}_{q}^{n}, w_{\mathbb{P}}\right)$ acts transitively on each $\mathbb{P}$-sphere $S_{\mathbb{P}}(r)=\{x \in$ $\left.\mathbb{F}_{q}^{n} \mid w_{\mathbb{P}}(x)=r\right\}$ for $0 \leq r \leq n$.

$(1) \Leftrightarrow(2)$ is shown in [8]. (1) $\Rightarrow(3)$ is proved in [5] and [7], and $(3) \Rightarrow(1)$ in [7]. Finally, $(1) \Rightarrow(4)$ is verified in [5] and $(4) \Rightarrow(1)$ in [6]. Here we remark that the proofs for $(1) \Rightarrow(3)$ were found in the form of Macwilliams-type identities by applying the discrete Poisson summation formula to suitable $\mathbb{P}$-weight enumerators (cf. [5-7]).

Let us now pay our attention to the equivalence $(1) \Leftrightarrow(2)$ in Theorem A. We will denote the association scheme $\left(\mathbb{F}_{q}^{n},\left\{R_{i}\right\}_{i=0}^{n}\right)$, with $(x, y) \in R_{i} \Leftrightarrow$ $d_{\mathbb{P}_{0}}(x, y)=i\left(\mathbb{P}_{0}=n_{1} \mathbf{1} \oplus n_{2} \mathbf{1} \oplus \cdots \oplus n_{t} \mathbf{1}\right)$, by $H\left(n_{1}, \ldots, n_{t} ; q\right)$, which is what we call a weak Hamming scheme. Note that this becomes the usual Hamming scheme when $t=1$. Recall the following theorem of Delsarte [3], which is usually called generalized MacWilliams identity.

Theorem B. Let $Y$ be an additive code of the translation association scheme $\mathfrak{X}=\left(X,\left\{R_{i}\right\}_{i=0}^{n}\right)$. Then

$$
\left(a_{j}\left(Y^{\circ}\right)\right)_{j=0}^{n}=\frac{1}{|Y|}\left(a_{i}(Y)\right)_{i=0}^{n}\left(q_{i j}\right) .
$$

Here $Y$ is just a subgroup of $X, Y^{\circ}$ is the additive code of the dual scheme $\mathfrak{X}^{*}=\left(X^{*},\left\{R_{i}^{*}\right\}_{i=0}^{n}\right)$ of $\mathfrak{X}$, given by

$$
\begin{aligned}
Y^{\circ} & =\left\{\chi \in X^{*} \mid \chi(x)=1 \text { for all } x \in Y\right\}, \\
a_{i}(Y) & =\left|\left\{y \in Y \mid(0, y) \in R_{i}\right\}\right|, \\
a_{i}\left(Y^{\circ}\right) & =\left|\left\{\chi \in Y^{\circ} \mid(1, \chi) \in R_{i}^{*}\right\}\right|,
\end{aligned}
$$

and $\left(q_{i j}\right)$ is the $Q$-matrix of the scheme $\mathfrak{X}$.

An immediate consequence of Theorem B is the classical MacWilliams identity which can be expressed in weight-enumerator-free form as: for any linear code $\mathcal{C} \subseteq \mathbb{F}_{q}^{n}$,

$$
\left(a_{j}\left(\mathcal{C}^{\perp}\right)\right)_{j=0}^{n}=\frac{1}{|\mathcal{C}|}\left(a_{i}(\mathcal{C})\right)_{i=0}^{n}\left(p_{j}(i)\right)
$$

where $p_{j}(x)=p_{j}(x ; n, q)$ is the Krawtchouk polynomial defined by

$$
p_{j}(x)=\sum_{l=0}^{j}(-1)^{l}(q-1)^{j-l}\left(\begin{array}{l}
x \\
l
\end{array}\right)\left(\begin{array}{c}
n-x \\
j-l
\end{array}\right)(0 \leq j \leq n) .
$$


Likewise, we would have yet another way of obtaining "MacWilliams-type identity" if we could find the $Q$-matrix for the scheme $H\left(n_{1}, \ldots, n_{t} ; q\right)$, which is an example of weak metric schemes, i.e., a finite wreath product of metric schemes (cf. [1], [9]).

Indeed, we observe that, for $i=1, \ldots, t, 1 \leq i_{0} \leq n_{i}$, or $i=1, i_{0}=0$,

$$
d_{\mathbb{P}_{0}}(x, y)=n_{1}+\cdots+n_{i-1}+i_{0} \Leftrightarrow x_{i+1}=y_{i+1}, \ldots, x_{t}=y_{t}, d_{H}\left(x_{i}, y_{i}\right)=i_{0},
$$

where $d_{H}$ is the Hamming metric. So identifying $\mathbb{F}_{q}^{n}$ with $\mathbb{F}_{q}^{n_{1}} \times \cdots \times \mathbb{F}_{q}^{n_{t}}$ by writing the elements $x \in \mathbb{F}_{q}^{n}$ as blocks of coordinates $x=\left(x_{1}, \ldots, x_{t}\right) \in$ $\mathbb{F}_{q}^{n_{1}} \times \cdots \times \mathbb{F}_{q}^{n_{t}}$, we see that $H\left(n_{1}, \ldots, n_{t} ; q\right)=H\left(n_{1}, q\right) \imath \cdots \imath H\left(n_{t}, q\right)$ is nothing but the wreath product of the Hamming schemes $H\left(n_{1}, q\right), \ldots, H\left(n_{t}, q\right)$, which are metric schemes as is well-known. This motivates our study of weak metric and weak cometric schemes. In particular, this will contribute to better understanding of the important weak Hamming scheme $H\left(n_{1}, \ldots, n_{t} ; q\right)$ (cf. Theorem A above).

This paper is organized as follows. In Section 2, we will fix some notations that will be used throughout this paper. In Section 3, the notion of weak metric schemes is introduced as a finite wreath product of metric schemes. Several equivalent conditions for being weak metric schemes are presented in Theorem 2. These include the conditions on the shape of the "first intersection matrices for each level", some modified polynomial relations on the adjacency matrices for each level and some modified polynomial relations on the $p$-numbers for each level. In Section 4 , for schemes given as a finite wreath product of (not necessarily metric) symmetric association schemes, some of their parameters are determined. But, for Proposition 9(b), Lemma 10 and Theorem 11, we assume that the schemes are weak metric schemes. Note that, for weak metric schemes, Theorem 7(a) completely determines the first intersection matrices for each level, whereas Theorem 2 (b) gives only information about the shapes of those ones. Theorem 11 is an analogue of [2, Theorem 1.3, p. 197 ]. However, proving that requires considerably more work. In Section 5, the notion of weak cometric schemes, which is dual to weak metric schemes, is introduced and some equivalent conditions for being cometric schemes are presented. Finally, in Section 6, we will give an example illustrating modified polynomial relations in the case of a weak Hamming scheme.

\section{Preliminaries and notations}

Let $n_{1}, \ldots, n_{t}$ be positive integers with $n=n_{1}+\cdots+n_{t}$. For each $i=$ $1,2, \ldots, t$, let $\mathfrak{X}^{(i)}=\left(X_{i},\left\{R_{j}^{(i)}\right\}_{j=0}^{n_{i}}\right)$ be a symmetric association scheme. Here we will always assume that the relations of $\mathfrak{X}^{(i)}$ are ordered as $R_{0}^{(i)}, R_{1}^{(i)}, \ldots$, $R_{n_{i}}^{(i)}$. Then $\mathfrak{X}=\mathfrak{X}^{(1)} \prec \cdots \prec \mathfrak{X}^{(t)}=\left(X=X_{1} \times \cdots \times X_{t},\left\{R_{j}\right\}_{j=0}^{n}\right)$ is the wreath product of $\mathfrak{X}^{(1)}, \mathfrak{X}^{(2)}, \ldots, \mathfrak{X}^{(t)}$, so that, for $\left(x_{1}, \ldots, x_{t}\right),\left(y_{1}, \ldots, y_{t}\right) \in X$,

$$
(x, y) \in R_{\underline{n_{i-1}}+i_{0}} \Leftrightarrow x_{i+1}=y_{i+1}, \ldots, x_{t}=y_{t} \text { and }\left(x_{i}, y_{i}\right) \in R_{i_{0}}^{(i)}
$$


for $i=1, \ldots, t, 1 \leq i_{0} \leq n_{i}$, or $i=1, i_{0}=0$ (cf. [1], [9]). Here we will always assume that the relations of $\mathfrak{X}$ are ordered as $R_{0}, R_{1}, \ldots, R_{n}$.

For each $i=1, \ldots, t$, let $\Gamma_{i}=\left(X_{i}, R_{1}^{(i)}\right)$ be the graph with the distance function $\partial_{i}$. Then, for $x=\left(x_{1}, \ldots, x_{t}\right), y=\left(y_{1}, \ldots, y_{t}\right) \in X$, we define

(1) $d(x, y)=\left\{\begin{array}{cl}0, & \text { if } x=y, \\ \underline{n_{i-1}}+\partial_{i}\left(x_{i}, y_{i}\right), & \text { if } x_{i} \neq y_{i} \text { and } x_{i+1}=y_{i+1}, \ldots, x_{t}=y_{t},\end{array}\right.$

where $\underline{n_{i-1}}=n_{1}+\cdots+n_{i-1}$. Then $d$ is a distance function. For this, we only need to check $d(x, y) \leq d(x, z)+d(z, y)$ for $x \neq y$. Assume $x_{i} \neq y_{i}, x_{i+1}=$ $y_{i+1}, \ldots, x_{t}=y_{t}$. Then $x_{i} \neq z_{i}$ or $z_{i} \neq y_{i}$, so that

$$
\begin{aligned}
d(x, z)+d(z, y) & \geq \underline{n_{i-1}}+\partial_{i}\left(x_{i}, z_{i}\right)+\partial_{i}\left(z_{i}, y_{i}\right) \\
& \geq \underline{n_{i-1}}+\partial_{i}\left(x_{i}, y_{i}\right) \\
& =d(x, y) .
\end{aligned}
$$

Remark 1. $(x, y) \in R_{\underline{n_{i-1}}}+1 \Leftrightarrow d(x, y)=\underline{n_{i-1}}+1(i=1, \ldots, t)$.

For elementary facts about association schemes, one is referred to [2] and [3]. Throughout this paper, the following notations will be used.

- $n_{1}, \ldots, n_{t}$ positive integers with $n_{1}+\cdots+n_{t}=n, n_{1}+\cdots+n_{i}=\underline{n_{i}}$ $(1 \leq i \leq t), \underline{n_{0}}=0, n_{t}+\cdots+n_{i}=\overline{n_{i}}(1 \leq i \leq t), \overline{n_{t+1}}=0$.

- $\left.\mathfrak{X}^{(i)}=\left(X_{i}, \overline{\{R}_{j}^{(i)}\right\}_{j=0}^{n_{i}}\right)$ a symmetric association scheme with a fixed ordering $R_{0}^{(i)}, R_{1}^{(i)}, \ldots, R_{n_{i}}^{(i)}$ of relations $(i=1, \ldots, t)$.

- Some of the parameters of $\mathfrak{X}^{(i)}$ used are: valencies $v_{0}^{(i)}=1, v_{1}^{(i)}, \ldots$, $v_{n_{i}}^{(i)}$, multiplicities $m_{0}^{(i)}=1, m_{1}^{(i)}, \ldots, m_{n_{i}}^{(i)}$, intersection numbers $p_{j l}^{(i) k}$, Krein parameters $q_{j l}^{(i) k}$, the adjacency matrices $A_{0}^{(i)}=I, A_{1}^{(i)}, \ldots, A_{n_{i}}^{(i)}$, the irreducible idempotents $E_{0}^{(i)}=\left|X_{i}\right|^{-1} J, E_{1}^{(i)}, \ldots, E_{n_{i}}^{(i)}$, the first intersection matrix $L_{1}^{(i)}=\left(p_{1 l}^{(i) k}\right)_{0 \leq k, l} \leq n_{i}$, the first dual intersection matrix $M_{1}^{(i)}=\left(q_{1 l}^{(i) k}\right)_{0 \leq k, l \leq n_{i}}$.

- $\mathfrak{X}=\left(X=X_{1} \times \cdots \times X_{t},\left\{R_{j}\right\}_{j=0}^{n}\right)$ the wreath product $\mathfrak{X}=\mathfrak{X}^{(1)}\left\{\cdots \cdot \mathfrak{X}^{(t)}\right.$ of $\mathfrak{X}^{(1)}, \ldots, \mathfrak{X}^{(t)}$, with a fixed ordering $R_{0}, R_{1}, \ldots, R_{n}$ of relations, so that $(x, y) \in R_{\underline{n_{i-1}}+i_{0}} \Leftrightarrow x_{i+1}=y_{i+1}, \ldots, x_{t}=y_{t}$ and $\left(x_{i}, y_{i}\right) \in R_{i_{0}}^{(i)}$ for $i=1, \ldots, t, \overline{1 \leq} i_{0} \leq n_{i}$, or $i=1, i_{0}=0$.

- Some of the parameters of $\mathfrak{X}$ used are: $p$-numbers $p_{j l}, q$-numbers $q_{j l}$, intersection numbers $p_{j l}^{k}$, Krein parameters $q_{j l}^{k}$, valencies $v_{0}=$ $1, v_{1}, \ldots, v_{n}$, multiplicities $m_{0}=1, m_{1}, \ldots, m_{n}$, the adjacency matrices $A_{0}, A_{1}, \ldots, A_{n}$, the irreducible idempotents $E_{0}, E_{1}, \ldots, E_{n}$, intersection matrices $L_{j}=\left(p_{j l}^{k}\right)_{0 \leq k, l \leq n}$, dual intersection matrices $M_{j}=$ $\left(q_{j l}^{k}\right)_{0 \leq k, l \leq n}$.

- Further notations for the parameters of $\mathfrak{X}$ are:

$\theta_{j}^{(i)}=p_{j, \underline{n_{i-1}}+1}, v^{(i)}=v_{n_{i-1}+1}\left(v^{(i)}=\theta_{0}^{(i)}\right)$,

$\omega_{j}^{(i)}=q_{j}, \overline{n_{i+1}}+1, m^{(i)}=\overline{m_{\overline{n_{i+1}}+1}}\left(m^{(i)}=\omega_{0}^{(i)}\right)$, 
$L^{(i)}=\left(p_{\underline{n_{i-1}}+1, l}^{k}\right)_{0 \leq k, l \leq n}$ the "first intersection matrix of level $i$ ",

$M^{(i)}=\left(\overline{q_{\overline{n_{i+1}}}^{k}+1, l}\right)_{0 \leq k, l \leq n}$ the "first dual intersection matrix of level $i "$

$\left[L^{(i)}\right]=\left(p_{n_{i-1}+1, l}^{k}\right)_{n_{i-1} \leq k, l \leq \underline{n_{i}}}$ a submatrix of $L^{(i)}$,

$\left[M^{(i)}\right]=\left(\overline{q_{\overline{n_{i+1}}}^{k}+1}, l\right) \overline{\overline{n_{i+1}}} \leq k, l \leq \overline{n_{i}}$ a submatrix of $M^{(i)}$.

- $\Gamma_{i}=\left(X_{i}, R_{1}^{(i)}\right)$ the graph with distance function $\partial_{i}, d$ the function on $X$ defined by (1).

\section{Weak metric schemes}

Let $\mathfrak{X}=\left(X=X_{1} \times \cdots \times X_{t},\left\{R_{j}\right\}_{j=0}^{n}\right)=\mathfrak{X}^{(1)} \prec \cdots \curlywedge \mathfrak{X}^{(t)}$ be the symmetric association scheme which is given as the wreath product of the symmetric association schemes $\mathfrak{X}^{(i)}=\left(X_{i},\left\{R_{j}^{(i)}\right\}_{j=0}^{n_{i}}\right)$ (cf. Section 2). Then it will be called a weak metric scheme if $\mathfrak{X}$ satisfies further the following equivalent conditions.

Theorem 2. The following are equivalent.

(a) $\Gamma_{i}=\left(X_{i}, R_{1}^{(i)}\right)$ is distance-regular for $i=1, \ldots, t$, and

$$
(x, y) \in R_{j} \Leftrightarrow d(x, y)=j \text { for } j=0, \ldots, n .
$$

(b) For $i=1, \ldots, t$, we have the following:

(i) The submatrix $\left[L^{(i)}\right]=\left(p_{\underline{n_{i-1}}+1}^{k}, l\right)_{n_{n_{i-1}} \leq k}, l \leq \underline{n_{i}}$ of

$$
L^{(i)}=\left(p_{\underline{n_{i-1}}}^{k}+1, l\right)_{0 \leq k, l \leq n}
$$

is a tridiagonal matrix with nonzero off-diagonal entries.

Moreover, $L^{(i)}$ has the following entries:

(ii) $\left(p_{n_{i-1}+1, l}^{k}\right)_{\underline{n_{i}}+1 \leq k, l \leq n}=v^{(i)} I$,

(iii) $p_{\underline{n_{i-1}}+1}^{k}, \underline{n_{i-1}+1}=v^{(i)}$ for $0 \leq k \leq \underline{n_{i-1}}$,

(iv) $p_{\frac{n_{i-1}}{n_{i-1}}+1, l}=v_{l}$ for $0 \leq l \leq \underline{n_{i-1}}$,

(v) All the other entries not appearing in (i)-(iv) are zeros.

(c) There are polynomials with real coefficients $\psi_{0}^{(1)}(x)$ of degree $0, \psi_{k}^{(i)}(x)$ of degree $k$ for $i=1, \ldots, t, 1 \leq k \leq n_{i}$, with $\psi_{k}^{(i)}(0)=0(2 \leq i \leq t)$, and real numbers $\alpha_{0}^{(1)}=\cdots=\alpha_{n_{1}}^{(1)}=0, \alpha_{1}^{(2)}, \ldots, \alpha_{n_{2}}^{(2)}, \ldots, \alpha_{1}^{(t)}, \ldots, \alpha_{n_{t}}^{(t)}$ such that

$$
A_{\underline{n_{i-1}}}+i_{0}=\psi_{i_{0}}^{(i)}\left(A_{\underline{n_{i-1}}+1}\right)+\alpha_{i_{0}}^{(i)}\left(A_{0}+\cdots+A_{\underline{n_{i-1}}}\right)
$$

for $i=1, \ldots, t, 1 \leq i_{0} \leq n_{i}$, or $i=1, i_{0}=0$.

(d) p-numbers satisfy

$$
p_{j,} \underline{n_{i-1}}+i_{0}=\psi_{i_{0}}^{(i)}\left(\theta_{j}^{(i)}\right)+\alpha_{i_{0}}^{(i)}\left(p_{j 0}+\cdots+p_{j, \underline{n_{i-1}}}\right)(0 \leq j \leq n),
$$

where $\psi_{i_{0}}^{(i)}$ 's and $\alpha_{i_{0}}^{(i)}$ 's are the same as in $(\mathrm{c})$, and $\theta_{j}^{(i)}=p_{j,} \underline{n_{i-1}}+1$.

(e) $\mathfrak{X}^{(i)}=\left(X_{i},\left\{R_{j}^{(i)}\right\}_{j=0}^{n_{i}}\right)$ is a metric scheme for $i=1, \ldots, t$. 
Proof. We first note the following: for $i=1, \ldots, t, 1 \leq i_{0} \leq n_{i}$, or $i=1, i_{0}=0$,

$$
A_{\underline{n_{i-1}}+i_{0}} A_{l}=v_{l} A_{\underline{n_{i-1}}}+i_{0} \text { for } 0 \leq l \leq \underline{n_{i-1}} .
$$

For this, we need to see: for $1 \leq j<i, 1 \leq k \leq n_{j}$, or $j=1, k=0$,

$$
A_{\underline{n_{i-1}}+i_{0}} A_{\underline{n_{j-1}+k}}=v_{\underline{n_{j-1}}+k} A_{\underline{n_{i-1}}+i_{0}} .
$$

Let $(x, y) \in R_{m}$. If $(x, z) \in R_{\underline{n_{i-1}}+i_{0}},(z, y) \in R_{n_{j-1}+k}$ for some $z \in X$, then $(x, y) \in R_{\underline{n_{i-1}}}+i_{0}$, and hence $m=\underline{n_{i-1}}+i_{0} . \overline{\text { On }}$ the other hand, if $(x, y) \in R_{\underline{n_{i-1}}+i_{0}}$, then

$$
\begin{aligned}
& \left\{z \in X \mid(x, z) \in R_{\underline{n_{i-1}}+i_{0}},(z, y) \in R_{\underline{n_{j-1}}+k}\right\} \\
= & \left\{z \in X \mid(z, y) \in R_{\underline{n_{j-1}}+k}\right\} .
\end{aligned}
$$

This shows (4).

(a) $\Rightarrow$ (b) (i) Let $k=\underline{n_{i-1}}+k_{0}, l=\underline{n_{i-1}}+l_{0}$, with $0 \leq k_{0}, l_{0} \leq n_{i}$. Suppose $p_{n_{i-1}+1, l}^{k} \neq 0$. Then $d(x, y)=k, d(x, z)=\underline{n}_{i-1}+1, d(z, y)=l$ for some $x, y, z$. Then $\partial_{i}\left(x_{i}, y_{i}\right)=k_{0}, \partial_{i}\left(x_{i}, z_{i}\right)=1, \partial_{i}\left(z_{i}, \overline{y_{i}}\right)=l_{0}$. So $\left|k_{0}-l_{0}\right| \leq 1$, and hence $|k-l| \leq 1$. Thus $p_{\underline{n_{i-1}}+1, l}^{k}=0$, if $|k-l| \geq 2$, with $\underline{n_{i-1}} \leq k, l \leq \underline{n_{i}}$. Also, $p_{n_{i-1}+1, l}^{k} \neq 0$, if $\mid k-\overline{l \mid}=1$. For any $x_{i}, y_{i} \in X_{i}$, with $\partial_{i}\left(x_{i}, y_{i}\right)=k_{0}(\geq 1)$, there is a path $x_{i}=z_{0}^{(i)}, z_{1}^{(i)}, \ldots, z_{k_{0}}^{(i)}=y_{i}$. Then $\partial_{i}\left(x_{i}, z_{1}^{(i)}\right)=1, \partial_{i}\left(z_{1}^{(i)}, y_{i}\right)=k_{0}-1$. Choose any points $x_{j} \in X_{j}$ for all $j \neq i$, and set

$$
\begin{aligned}
& x=\left(x_{1}, \ldots, x_{i-1}, x_{i}, x_{i+1}, \ldots, x_{t}\right), \\
& y=\left(x_{1}, \ldots, x_{i-1}, y_{i}, x_{i+1}, \ldots, x_{t}\right), \\
& z=\left(x_{1}, \ldots, x_{i-1}, z_{1}^{(i)}, x_{i+1}, \ldots, x_{t}\right) .
\end{aligned}
$$

Then $d(x, y)=\underline{n_{i-1}}+k_{0}, d(x, z)=\underline{n_{i-1}}+1, d(z, y)=\underline{n_{i-1}}+k_{0}-1$. So $p_{\underline{n_{i-1}}+1, k-1}^{k} \neq 0 \overline{\text { for } \underline{n}_{i-1}}+1 \leq k \leq \underline{n_{i}}$. Similarly, one shows that $p_{\underline{n_{i-1}}+1, k+1}^{k} \neq$ $0 \overline{\text { for }} n_{i-1} \leq k \leq \underline{n_{i}}-1$.

(ii) Let $\underline{n_{i}}+1 \leq \bar{l} \leq n$. For any $x, y$ with $d(x, y)=l$,

$$
\begin{aligned}
p_{\underline{n_{i-1}}+1, l}^{l} & =\left|\left\{z \mid d(x, z)=\underline{n_{i-1}}+1, d(z, y)=l\right\}\right| \\
& =\left|\left\{z \mid d(x, z)=\underline{n_{i-1}}+1\right\}\right| \\
& =v^{(i)} .
\end{aligned}
$$

Indeed, if $l=\underline{n_{j}}+l_{0}\left(1 \leq l_{0} \leq n_{j+1}\right)$ for some $j$ with $i \leq j \leq t-1$, then, for $z$ with $d(x \overline{, z})=\underline{n_{i-1}}+1, x_{j+1}=z_{j+1}, \partial_{j+1}\left(x_{j+1}, y_{j+1}\right)=l_{0}, z_{j+2}=$ $y_{j+2}, \ldots, z_{t}=y_{t}$, and hence $d(z, y)=l$ is automatic. In the same manner, one shows $p_{n_{i-1}+1, l}^{k}=0$ for all $k, l$ with $\underline{n_{i}}+1 \leq k, l \leq n, k \neq l$.

(iii), (iv) These can be shown similarly to the proof of (ii).

(v) As $p_{\underline{n_{i-1}}+1, l}^{k}=0 \Leftrightarrow p_{\underline{n_{i-1}}+1, k}^{l}=0$, we only need to see:

$$
p_{\underline{n_{i-1}}}^{k}+1, l=0 \text { for } 0 \leq k \leq n\left(k \neq \underline{n_{i-1}}+1\right), 0 \leq l \leq \underline{n_{i-1}},
$$


and

$$
p_{\underline{n_{i-1}}}^{k}+1, l=0 \text { for } \underline{n_{i-1}}+1 \leq k \leq \underline{n_{i}}, \underline{n_{i}}+1 \leq l \leq n .
$$

Both of these are easy to see.

(b) $\Rightarrow$ (a) First, we show (2) by induction on $j$. It is clearly true for $j=$ 0 . Assume that $k \geq 1$ and the assertion holds for all $j \leq k-1$. Let $k=$ $n_{i-1}+k_{0}$ for some $i, k_{0}$ with $1 \leq i \leq t, 1 \leq k_{0} \leq n_{i}$. Let $(x, y) \in R_{k}$. As $\overline{p_{n_{i-1}}^{k}}+1, k-1 \neq 0,(x, z) \in R_{\underline{n_{i-1}}+1},(z, y) \in R_{k-1}$ for some $z$. By induction hypothesis $d(z, y)=k-1$, and $d(x, z)=\underline{n_{i-1}}+1$, by Remark 1 . Thus

$$
\begin{aligned}
& \partial_{i}\left(z_{i}, y_{i}\right)=k_{0}-1, z_{i+1}=y_{i+1}, \ldots, z_{t}=y_{t} \\
& \partial_{i}\left(x_{i}, z_{i}\right)=1, x_{i+1}=z_{i+1}, \ldots, x_{t}=z_{t} .
\end{aligned}
$$

So $\partial_{i}\left(x_{i}, y_{i}\right) \leq k_{0}, x_{i+1}=y_{i+1}, \ldots, x_{t}=y_{t}$, and hence $d(x, y) \leq k$. Also, we have $d(x, y) \geq k$, since otherwise we would have $(x, y) \in R_{j}$ for some $j \leq k-1$, by induction hypothesis. Conversely, assume that $d(x, y)=k$. Here, in view of Remark 1, we may let $d(x, y)=k=\underline{n_{i-1}}+k_{0}$ for some $i$ and $k_{0}$ with $2 \leq k_{0} \leq n_{i}$. Then we must have $(x, y) \in \overline{R_{j}}$ for some $j \geq k$. We now exclude the possibility that $(x, y) \in R_{j}$ for some $j \geq k+1$. Assume, on the contrary, that $(x, y) \in R_{j}$ for some $j \geq k+1$. Then there is a path $x_{i}=z_{0}^{(i)}, z_{1}^{(i)}, \ldots, z_{k_{0}-1}^{(i)}, z_{k_{0}}^{(i)}=y_{i}$, and $x_{i+1}=y_{i+1}, \ldots, x_{t}=y_{t}$. Put

$$
z=\left(x_{1}, \ldots, x_{i-1}, z_{k_{0}-1}^{(i)}, x_{i+1}, \ldots, x_{t}\right) .
$$

Then $d(y, z)=\underline{n_{i-1}}+1 \Rightarrow(y, z) \in R_{n_{i-1}+1}$, by Remark 1 , and $d(z, x)=k-1 \Rightarrow$

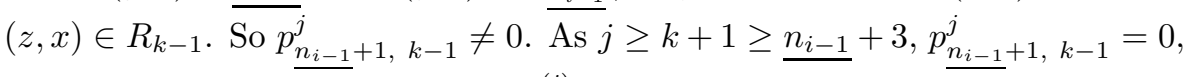
by our assumption on the matrix $L^{(i)}$. This is a contradiction.

Next, we show that each $\Gamma_{i}$ is distance-regular. Let $x_{i}, y_{i}, x_{i}^{\prime}, y_{i}^{\prime} \in X_{i}$, with $\partial_{i}\left(x_{i}, y_{i}\right)=\partial_{i}\left(x_{i}^{\prime}, y_{i}^{\prime}\right)=k_{0}\left(0 \leq k_{0} \leq n_{i}\right)$. Then we must show:

$$
\begin{gathered}
\left|\left\{u_{i} \in X_{i} \mid \partial_{i}\left(x_{i}, u_{i}\right)=l_{0}, \partial_{i}\left(u_{i}, y_{i}\right)=m_{0}\right\}\right| \\
=\left|\left\{u_{i} \in X_{i} \mid \partial_{i}\left(x_{i}^{\prime}, u_{i}\right)=l_{0}, \partial_{i}\left(u_{i}, y_{i}^{\prime}\right)=m_{0}\right\}\right|
\end{gathered}
$$

for all $l_{0}, m_{0}$ with $0 \leq l_{0}, m_{0} \leq n_{i}$. This is clearly true for $l_{0}=0$ or $m_{0}=0$. Thus we assume $l_{0}, m_{0} \geq 1$. Choose $z_{j} \in X_{j}$ for all $j \neq i$, and set

$$
\begin{aligned}
& \tilde{x}=\left(z_{1}, \ldots, z_{i-1}, x_{i}, z_{i+1}, \ldots, z_{t}\right), \tilde{y}=\left(z_{1}, \ldots, z_{i-1}, y_{i}, z_{i+1}, \ldots, z_{t}\right), \\
& \tilde{x}^{\prime}=\left(z_{1}, \ldots, z_{i-1}, x_{i}^{\prime}, z_{i+1}, \ldots, z_{t}\right), \tilde{y}^{\prime}=\left(z_{1}, \ldots, z_{i-1}, y_{i}^{\prime}, z_{i+1}, \ldots, z_{t}\right) .
\end{aligned}
$$

Then $d(\tilde{x}, \tilde{y})=d\left(\tilde{x}^{\prime}, \tilde{y}^{\prime}\right)$. By $(2)$, we have:

$$
\begin{gathered}
\left|\left\{u \in X \mid d(\tilde{x}, u)=\underline{n_{i-1}}+l_{0}, d(u, \tilde{y})=\underline{n_{i-1}}+m_{0}\right\}\right| \\
=\left|\left\{u \in X \mid d\left(\tilde{x}^{\prime}, u\right)=\underline{n_{i-1}}+l_{0}, d\left(u, \tilde{y}^{\prime}\right)=\underline{n_{i-1}}+m_{0}\right\}\right|,
\end{gathered}
$$

which implies

$$
\left|X_{1}\right| \cdots\left|X_{i-1}\right| \cdot\left|\left\{u_{i} \in X_{i} \mid \partial_{i}\left(x_{i}, u_{i}\right)=l_{0}, \partial_{i}\left(u_{i}, y_{i}\right)=m_{0}\right\}\right|
$$




$$
=\left|X_{1}\right| \cdots\left|X_{i-1}\right| \cdot\left|\left\{u_{i} \in X_{i} \mid \partial_{i}\left(x_{i}^{\prime}, u_{i}\right)=l_{0}, \partial_{i}\left(u_{i}, y_{i}^{\prime}\right)=m_{0}\right\}\right| .
$$

So we get $(6)$.

(b) $\Rightarrow$ (c) Here we denote the entries of the submatrix $\left[L^{(i)}\right]$ of $L^{(i)}$ just as in $(12)$ for $i=1, \ldots, t$. Define the polynomials $\psi_{i}^{(1)}(x)$ of degree $i\left(0 \leq i \leq n_{1}\right)$ recursively by:

$$
\begin{aligned}
\psi_{0}^{(1)}(x) & =1, \psi_{1}^{(1)}(x)=x, \\
x \psi_{i}^{(1)}(x) & =b_{i-1}^{(1)} \psi_{i-1}^{(1)}(x)+a_{i}^{(1)} \psi_{i}^{(1)}(x)+c_{i+1}^{(1)} \psi_{i+1}^{(1)}(x)(i \geq 1) .
\end{aligned}
$$

Also, define

$$
\alpha_{0}^{(1)}=\alpha_{1}^{(1)}=\cdots=\alpha_{n_{1}}^{(1)}=0 .
$$

Then

$$
A_{i_{0}}=\psi_{i_{0}}^{(1)}\left(A_{1}\right)+\alpha_{i_{0}}^{(1)} A_{0} \text { for } 0 \leq i_{0} \leq n_{1} .
$$

Let $i(2 \leq i \leq t)$ be fixed. Define

$$
\psi_{1}^{(i)}(x)=x, \alpha_{1}^{(i)}=0 .
$$

Then

$$
A_{\underline{n_{i-1}}+1}=\psi_{1}^{(i)}\left(A_{\underline{n_{i-1}}+1}\right)+\alpha_{1}^{(i)}\left(A_{0}+\cdots+A_{\underline{n_{i-1}}}\right) .
$$

Also, if we define

$$
\psi_{2}^{(i)}(x)=c_{2}^{(i)^{-1}}\left(x^{2}-a_{1}^{(i)} x\right), \alpha_{2}^{(i)}=-c_{2}^{(i)^{-1}} v^{(i)},
$$

then

$$
A_{\underline{n_{i-1}}+2}=\psi_{2}^{(i)}\left(A_{\underline{n_{i-1}}+1}\right)+\alpha_{2}^{(i)}\left(A_{0}+\cdots+A_{\underline{n_{i-1}}}\right) .
$$

Assume now that $\psi_{1}^{(i)}, \ldots, \psi_{i_{0}}^{(i)}, \alpha_{1}^{(i)}, \ldots, \alpha_{i_{0}}^{(i)}\left(2 \leq i_{0}<n_{i}\right)$ are defined so that

$$
A_{\underline{n_{i-1}}}+j_{0}=\psi_{j_{0}}^{(i)}\left(\underline{A_{n_{i-1}}+1}\right)+\alpha_{j_{0}}^{(i)}\left(A_{0}+\cdots+A_{\underline{n_{i-1}}}\right), \psi_{j_{0}}^{(i)}(0)=0
$$

holds for all $1 \leq j_{0} \leq i_{0}$. Define the polynomial $\psi_{i_{0}+1}^{(i)}(x)$ of degree $i_{0}+1$ by:

$$
\begin{aligned}
\psi_{i_{0}+1}^{(i)}(x)= & c_{i_{0}+1}^{(i)^{-1}}\left\{x \psi_{i_{0}}^{(i)}(x)+\alpha_{i_{0}}^{(i)}\left(v_{0}+\cdots+\underline{v_{n_{i-1}}}\right) x\right. \\
& \left.-b_{i_{0}-1}^{(i)} \psi_{i_{0}-1}^{(i)}(x)-a_{i_{0}}^{(i)} \psi_{i_{0}}^{(i)}(x)\right\},
\end{aligned}
$$

and a constant $\alpha_{i_{0}+1}^{(i)}$ by:

$$
\alpha_{i_{0}+1}^{(i)}=-c_{i_{0}+1}^{(i)^{-1}}\left(b_{i_{0}-1}^{(i)} \alpha_{i_{0}-1}^{(i)}+a_{i_{0}}^{(i)} \alpha_{i_{0}}^{(i)}\right) .
$$

Then

$$
A_{\underline{n_{i-1}}}+i_{0}+1=\psi_{i_{0}+1}^{(i)}\left(A_{\underline{n_{i-1}}+1}\right)+\alpha_{i_{0}+1}^{(i)}\left(A_{0}+\cdots+A_{\underline{n_{i-1}}}\right) .
$$

Observe here that $\psi_{i_{0}+1}^{(i)}(0)=0$. 
(c) $\Rightarrow$ (b) Here we must show the following: for $i=1, \ldots, t$,

$$
A_{\underline{n_{i-1}}+1} A_{l}=v_{l} A_{\underline{n_{i-1}}+1}\left(0 \leq l \leq \underline{n_{i-1}}\right),
$$

(8) $\quad A_{\underline{n_{i-1}+1}} A_{\underline{n_{i-1}+1}}=v^{(i)}\left(A_{0}+\cdots+A_{\underline{n_{i-1}}}\right)+a_{1}^{(i)} A_{\underline{n_{i-1}}+1}+c_{2}^{(i)} A_{\underline{n_{i-1}}+2}$ with $c_{2}^{(i)} \neq 0$,

$$
\begin{aligned}
& A_{\underline{n_{i-1}}+1} A_{\underline{n_{i-1}}+i_{0}}=b_{i_{0}-1}^{(i)} A_{\underline{n_{i-1}}+i_{0}-1}+a_{i_{0}}^{(i)} A_{\underline{n_{i-1}}+i_{0}} \\
& +c_{i_{0}+1}^{(i)} A_{\underline{n_{i-1}}+i_{0}+1}\left(2 \leq i_{0} \leq n_{i}\right)
\end{aligned}
$$

with $b_{i_{0}-1}^{(i)} \neq 0\left(2 \leq i_{0} \leq n_{i}\right), c_{i_{0}+1}^{(i)} \neq 0\left(2 \leq i_{0} \leq n_{i}-1\right), c_{n_{i}+1}^{(i)}=0$,

$$
A_{\underline{n_{i-1}}}+1 A_{l}=v^{(i)} A_{l}\left(\underline{n_{i}}+1 \leq l \leq n\right) \text {. }
$$

By (4), (7), and (10) are true. Next, we show (8) and (9). For $i=1$, we only need to show (9) for $1 \leq l \leq n_{1}$. By our assumption $A_{i_{0}}=\psi_{i_{0}}^{(1)}\left(A_{1}\right)$ for $0 \leq i_{0} \leq n_{1}$. Since $x \psi_{l}^{(1)}(x)$ is a linear combination of $\psi_{l+1}^{(1)}(x), \psi_{l}^{(1)}(x), \ldots$, $\psi_{0}^{(1)}(x), A_{1} A_{l}$ is a linear combination of $A_{l+1}, A_{l}, \ldots, A_{0}$. Clearly, the coefficient of $A_{l+1} \neq 0$. As $A_{1} A_{l}=\sum_{k=0}^{n} p_{1 l}^{k} A_{k}, p_{1 l}^{k}=0$ for $k \geq l+2$ and $p_{1 l}^{l+1} \neq 0$. Since $p_{1 l}^{k}=0 \Leftrightarrow p_{1 k}^{l}=0, p_{1 l}^{k}=0$ if $|k-l| \geq 2$, and $p_{1 l}^{k} \neq 0$ if $|k-l|=1$. Let $i \geq 2$. First, we show (9). By (3) and (4), for $1 \leq i_{0} \leq n_{i}$ we have:

$$
A_{\underline{n_{i-1}}+i_{0}} A_{\underline{n_{i-1}}+1}=\psi_{i_{0}}^{(i)}\left(A_{\underline{n_{i-1}}+1}\right) A_{\underline{n_{i-1}}+1}+\alpha_{i_{0}}^{(i)}\left(v_{0}+\cdots+\underline{v_{n_{i-1}}}\right) A_{\underline{n_{i-1}}+1} .
$$

As $\psi_{k}^{(i)}(0)=0\left(1 \leq k \leq n_{i}\right)$,

$$
\psi_{i_{0}}^{(i)}(x) x+\alpha_{i_{0}}^{(i)}\left(v_{0}+\cdots+v_{\underline{n_{i-1}}}\right) x=\sum_{k_{0}=1}^{i_{0}+1} \beta_{k_{0}} \psi_{k_{0}}^{(i)}(x)
$$

for some $\beta_{k_{0}}$ with $\beta_{i_{0}+1} \neq 0$. Thus, for $2 \leq i_{0} \leq n_{i}$,

$$
A_{\underline{n_{i-1}}}+i_{0} A_{\underline{n_{i-1}}+1}=\sum_{k_{0}=1}^{i_{0}+1} \beta_{k_{0}} A_{\underline{n_{i-1}}}+k_{0}-\sum_{k_{0}=1}^{i_{0}+1} \beta_{k_{0}} \alpha_{k_{0}}^{(i)}\left(A_{0}+\cdots+A_{\underline{n_{i-1}}}\right),
$$

by (3) and (11). Just as in the above argument, this implies that $p_{\underline{n_{i-1}}}^{n_{i-1}}+k_{0}, \underline{n_{i-1}}+l_{0}$ $=0$ for $\left|k_{0}-l_{0}\right| \geq 2$, and $p \underline{n_{n_{i-1}}}+1, \underline{n_{i-1}}+l_{0} \neq 0$ for $\left|k_{0}-l_{0}\right|=1$. Next, we show (8). By (3) with $i_{0}=2$,

$$
A_{\underline{n_{i-1}}+2}=a A_{\underline{n_{i-1}}+1}^{2}+b A_{\underline{n_{i-1}}+1}+\alpha \alpha_{2}^{(i)}\left(A_{0}+\cdots+A_{\underline{n_{i-1}}}\right)
$$

for some $a, b$ with $a \neq 0$. So

$$
A_{\underline{n_{i-1}}+1} A_{\underline{n_{i-1}}+1}=w\left(A_{0}+\cdots+A_{\underline{n_{i-1}}}\right)+a_{1}^{(i)} A_{\underline{n_{i-1}}+1}+c_{2}^{(i)} A_{\underline{n_{i-1}+2}}
$$


with $c_{2}^{(i)} \neq 0$, where we put

$$
w=-a^{-1} \alpha_{2}^{(i)}, a_{1}^{(i)}=-a^{-1} b, c_{2}^{(i)}=a^{-1} .
$$

It remains to see that $w=v^{(i)}$. For $0 \leq k \leq \underline{n_{i-1}}$, let $(x, y) \in R_{k}$. Then $x_{i}=y_{i}, \ldots, x_{t}=y_{t}$. Thus $p_{\underline{n_{i-1}}+1, \underline{n_{i-1}}}^{k}+1=v^{(i)}$, and hence $w=v^{(i)}$.

(c) $\Leftrightarrow$ (d) This is straightforward.

(a) $\Leftrightarrow$ (e) Note that (2) is equivalent to: for $i=1, \ldots, t$,

$$
\left(x_{i}, y_{i}\right) \in R_{j}^{(i)} \Leftrightarrow \partial_{i}\left(x_{i}, y_{i}\right)=j\left(0 \leq j \leq n_{i}\right) \quad \text { (cf. [2, Prop. 1.1, p. 189]). }
$$

Remark 3. (1) When $\mathfrak{X}$ is a weak metric scheme, for $i=1, \ldots, t$ the submatrix $\left[L^{(i)}\right]$ of $L^{(i)}$ will be denoted by:

$$
\left[L^{(i)}\right]=\left[\begin{array}{ccccc}
a_{0}^{(i)} & b_{0}^{(i)} & & & \\
c_{1}^{(i)} & a_{1}^{(i)} & b_{1}^{(i)} & & \\
& \ddots & \ddots & \ddots & \\
& & c_{n_{i}-1}^{(i)} & a_{n_{i}-1}^{(i)} & b_{n_{i}-1}^{(i)} \\
& & & c_{n_{i}}^{(i)} & a_{n_{i}}^{(i)}
\end{array}\right] .
$$

Observe here that $a_{0}^{(i)}=0, b_{0}^{(i)}=v^{(i)}, c_{1}^{(i)}=v_{n_{i-1}}$.

(2) Put

$\psi^{(1)}(x)=\psi_{0}^{(1)}(x)+\cdots+\psi_{n_{1}}^{(1)}(x), \psi^{(i)}(x)=\psi_{1}^{(i)}(x)+\cdots+\psi_{n_{i}}^{(i)}(x)(2 \leq i \leq t)$.

Then $\psi^{(i)}(x)$ is a polynomial of degree $n_{i}$ for $1 \leq i \leq t$. Using (3), one can show by induction on $i$ that, for $i=2, \ldots, t$,

$$
A_{0}+\cdots+A_{\underline{n_{i-1}}}=\sum_{k=1}^{i-1} c_{k}^{(i)} \psi^{(k)}\left(A_{\underline{n_{k-1}}+1}\right)
$$

where $c_{k}^{(i)}=\prod_{l=k+1}^{i-1}\left(1+\alpha_{1}^{(l)}+\cdots+\alpha_{n_{l}}^{(l)}\right)$ with the understanding that $c_{i-1}^{(i)}=1$.

(3) For $i=2, \ldots, t, 1 \leq i_{0} \leq n_{i}$,

$$
A_{\underline{n_{i-1}}+i_{0}}=\psi_{i_{0}}^{(i)}\left(A_{\underline{n_{i-1}}+1}\right)+\alpha_{i_{0}}^{(i)} \xi_{n_{i-1}+1}^{(i)}\left(A_{\underline{n_{i-2}}+1}\right),
$$

where $\xi_{n_{i-1}+1}^{(i)}(x)$ is a polynomial with real coefficients of degree $n_{i-1}+1$. Indeed, from (3) and (13), we have

$$
A_{\underline{n_{i-1}}}+i_{0}=\psi_{i_{0}}^{(i)}\left(A_{\underline{n_{i-1}}}+1\right)+\alpha_{i_{0}}^{(i)} \sum_{k=1}^{i-1} c_{k}^{(i)} \psi^{(k)}\left(A_{\underline{n_{k-1}}}+1\right) .
$$

Multiplying both sides of (14) by $A_{\underline{n_{i-2}}+1}$ and using (4), we get:

$$
\begin{aligned}
& v^{(i-1)} A_{\underline{n_{i-1}}+i_{0}} \\
= & v^{(i-1)} \psi_{i_{0}}^{(i)}\left(\underline{A_{n_{i-1}}}+1\right)
\end{aligned}
$$




$$
\begin{aligned}
& +\alpha_{i_{0}}^{(i)} \sum_{k=1}^{i-2} c_{k}^{(i)} \psi^{(k)}\left(A_{\underline{n_{k-1}}}+1\right) A_{\underline{n_{i-2}}+1}+\alpha_{i_{0}}^{(i)} \psi^{(i-1)}\left(A_{\underline{n_{i-2}}+1}\right) A_{\underline{n_{i-2}}}+1 \\
= & v^{(i-1)} \psi_{i_{0}}^{(i)}\left(A_{\underline{n_{i-1}}+1}\right) \\
& +\alpha_{i_{0}}^{(i)}\left\{\sum_{k=1}^{i-2} c_{k}^{(i)} \psi^{(k)}\left(v^{(k)}\right) A_{\underline{n_{i-2}}}+1\right. \\
& \left.+\psi^{(i-1)}\left(A_{\underline{n_{i-2}}+1}\right) A_{\underline{n_{i-2}}+1}\right\} .
\end{aligned}
$$

This implies

$$
A_{\underline{n_{i-1}}+i_{0}}=\psi_{i_{0}}^{(i)}\left(A_{\underline{n_{i-1}}+1}\right)+\alpha_{i_{0}}^{(i)} \xi_{n_{i-1}+1}^{(i)}\left(A_{\underline{n_{i-2}}}+1\right),
$$

where we put

$$
\xi_{n_{i-1}+1}^{(i)}(x)=v^{(i-1)^{-1}}\left\{\sum_{k=1}^{i-2} c_{k}^{(i)} \psi^{(k)}\left(v^{(k)}\right) x+\psi^{(i-1)}(x) x\right\} .
$$

Proposition 4. Let $\mathfrak{X}=\left(X=X_{1} \times \cdots \times X_{t},\left\{R_{j}\right\}_{j=0}^{n}\right)=\mathfrak{X}^{(1)} \prec \cdots \prec \mathfrak{X}^{(t)}$ be a weak metric scheme with $\mathfrak{X}^{(i)}=\left(X_{i},\left\{R_{j}^{(i)}\right\}_{j=0}^{n_{i}}\right)$. Let the entries of the submatrix $\left[L^{(i)}\right]$ of $L^{(i)}$ be as in (12). Then

(a)

$$
\begin{gathered}
a_{l}^{(1)}+b_{l}^{(1)}+c_{l}^{(1)}=v^{(1)} \text { for } i \geq 2, \\
a_{l}^{(i)}+b_{l}^{(i)}+c_{l}^{(i)}=\left\{\begin{array}{l}
v^{(i)}, 0 \leq l \leq n_{i} \text { and } l \neq 1, \\
v^{(i)}-\left(v_{0}+v_{1}+\cdots+v_{\underline{n_{i-1}}}-1\right), \quad l=1 .
\end{array}\right.
\end{gathered}
$$

Here $c_{0}^{(i)}=0, b_{n_{i}}^{(i)}=0$ for $i=1, \ldots, t$.

(b) $v_{n_{i-1}+i_{0}}=v^{(i)} b_{1}^{(i)} b_{2}^{(i)} \cdots b_{i_{0}-1}^{(i)} / c_{2}^{(i)} c_{3}^{(i)} \cdots c_{i_{0}}^{(i)}$ for $i=1, \ldots, t, 2 \leq i_{0} \leq n_{i}$.

(c) $\overline{v^{(i)}}=b_{0}^{(i)} \geq b_{1}^{(i)} \geq \cdots \geq b_{n_{i}-1}^{(i)}$ for $i=1, \ldots, t$.

(d) $v_{n_{i-1}}=c_{1}^{(i)} \leq c_{2}^{(i)} \leq \cdots \leq c_{n_{i}}^{(i)}$ for $i=1, \ldots, t$.

Proof. (a) The result follows from $\sum_{j=0}^{n} p_{\underline{n_{i-1}}+1, j}^{k}=v^{(i)}$, Theorem 2(b)(i), (iv), (v), and (12).

(b) Since $p_{i j}^{k} v_{k}=p_{i k}^{j} v_{j}, v_{\underline{n_{i-1}}+i_{0}} b_{i_{0}}^{(i)}=v_{\underline{n_{i-1}}+i_{0}+1} c_{i_{0}+1}^{(i)}$. So

$$
v^{(i)}=\frac{c_{2}^{(i)} v_{n_{i-1}+2}}{b_{1}^{(i)}}=\frac{c_{2}^{(i)} c_{3}^{(i)} v_{n_{i-1}+3}}{b_{1}^{(i)} b_{2}^{(i)}}=\cdots=\frac{c_{2}^{(i)} \cdots c_{i_{0}}^{(i)} v_{n_{i-1}+i_{0}}}{b_{1}^{(i)} b_{2}^{(i)} \cdots b_{i_{0}-1}^{(i)}} .
$$

(c) Let $x, y \in X$ with $d(x, y)=n_{i-1}+i_{0}\left(1 \leq i_{0} \leq n_{i}-1\right)$. Let $w$ be an element of $X$ such that $d(x, w)=\overline{=n_{i-1}}+1, d(w, y)=n_{i-1}+i_{0}+1$. As $\partial_{i}\left(x_{i}, y_{i}\right)=i_{0}$, we can choose $z_{i} \in X_{i}$, with $\partial_{i}\left(x_{i}, z_{i}\right)=i_{0}-\overline{-1}, \partial_{i}\left(z_{i}, y_{i}\right)=1$. Then $\partial_{i}\left(z_{i}, w_{i}\right) \leq i_{0}$. Also, $\partial_{i}\left(z_{i}, w_{i}\right) \geq i_{0}$, since otherwise we would have $\partial_{i}\left(w_{i}, y_{i}\right) \leq i_{0}$. We put $z=\left(x_{1}, \ldots, x_{i-2}, z_{i-1}, z_{i}, x_{i+1}, \ldots, x_{t}\right)$, where $z_{i-1} \in$ $X_{i-1}$ is an element with $\partial_{i-1}\left(x_{i-1}, z_{i-1}\right)=n_{i-1}$. Note here that such an $z_{i-1}$ can be chosen, since, if necessary, after replacing $(i-1)$ th component 
$x_{i-1}$ of $x$ we may assume $\left\{a_{i-1} \in X_{i-1} \mid \partial_{i-1}\left(x_{i-1}, a_{i-1}\right)=n_{i-1}\right\} \neq \varnothing$. Then $d(x, z)=\underline{n_{i-1}}+i_{0}-1$. Observe that this holds even for $i_{0}=1$. Also, $d(w, z)=\underline{n_{i-1}}+\overline{i_{0}}$. This shows that $b_{i_{0}}^{(i)} \leq b_{i_{0}-1}^{(i)}$ for $1 \leq i_{0} \leq n_{i}-1$.

(d) Let $\overline{x, z} \in X$, with $d(x, z)=n_{i-1}+i_{0}+1\left(1 \leq i_{0} \leq n_{i}-1\right)$. Choose $y_{i} \in$ $X_{i}$ with $\partial_{i}\left(x_{i}, y_{i}\right)=i_{0}, \partial_{i}\left(y_{i}, z_{i}\right)=\overline{1 \text {. Put }} y=\left(x_{1}, \ldots, x_{i-1}, y_{i}, x_{i+1}, \ldots, x_{t}\right)$. Then $d(x, y)=n_{i-1}+i_{0}$. Let $w$ be an element of $X$ such that $d(x, w)=n_{i-1}+1$, $d(w, y)=n_{i-1} \overline{+i_{0}}-1$. Just as in the proof of (c), we have $\partial_{i}\left(w_{i}, z_{i}\right) \overline{i_{0}}$, and hence $d\left(\overline{w, z)}=\underline{n_{i-1}}+i_{0}\right.$. This shows $c_{i_{0}}^{(i)} \leq c_{i_{0}+1}^{(i)}$ for $1 \leq i_{0} \leq n_{i}-1$.

\section{Parameters of weak metric schemes}

Let $\mathfrak{X}=\left(X=X_{1} \times \cdots \times X_{t},\left\{R_{j}\right\}_{j=0}^{n}\right)=\mathfrak{X}^{(1)} \imath \cdots \prec \mathfrak{X}^{(t)}$ be a symmetric association scheme which is given as the wreath product of the symmetric association schemes $\mathfrak{X}^{(i)}=\left(X_{i},\left\{R_{j}^{(i)}\right\}_{j=0}^{n_{i}}\right)$. Then we will determine some of the parameters of $\mathfrak{X}$, such as valencies, multiplicities, the adjacency matrices, the irreducible idempotents, $P$-matrix and $Q$-matrix. Further, we will determine the first intersection matrix for each level and the first dual intersection matrix for each level. Also, we will prove Theorem 11 which is an analogue of Theorem 1.3 in [2, p. 197].

In below, all the matrices in the $i$-th factor of tensor products are of size $\left|X_{i}\right|$ for $i=1, \ldots, t$, and accordingly $I$ and $J$ will denote respectively the identity and all-one matrices of various sizes.

Theorem 5. Let $\mathfrak{X}=\left(X=X_{1} \times \cdots \times X_{t},\left\{R_{j}\right\}_{j=0}^{n}\right)=\mathfrak{X}^{(1)} \imath \cdots \curlywedge \mathfrak{X}^{(t)}$ be a symmetric association scheme which is given as the wreath product of the symmetric association schemes $\mathfrak{X}^{(i)}=\left(X_{i},\left\{R_{j}^{(i)}\right\}_{j=0}^{n_{i}}\right)$. Then we have the following.

(a) $\left|X_{1}\right| \cdots\left|X_{i-1}\right| v_{i_{0}}^{(i)}=v_{\underline{n_{i-1}}+i_{0}}$ for $1 \leq i \leq t, 1 \leq i_{0} \leq n_{i}$, or $i=1$, $i_{0}=0$.

(b) $\lambda_{i}:=\sum \frac{n_{i}}{j=0} v_{j}=\left|X_{1}\right| \cdots\left|X_{i}\right|$ for $i=1, \ldots, t$.

(c) $\left|X_{t}\right| \cdots\left|X_{i+1}\right| m_{i_{0}}^{(i)}=m_{\overline{n_{i+1}}+i_{0}}$ for $1 \leq i \leq t, 1 \leq i_{0} \leq n_{i}$, or $i=t$, $i_{0}=0$.

(d) $\mu_{i}:=\sum_{j=0}^{\overline{n_{i}}} m_{j}=\left|X_{t}\right| \cdots\left|X_{i}\right|$ for $i=1, \ldots, t$.

(e)

$$
\begin{aligned}
A_{0} & =A_{0}^{(1)} \otimes I \otimes \cdots \otimes I=I, \quad A_{1}=A_{1}^{(1)} \otimes I \otimes \cdots \otimes I, \ldots, \\
A_{n_{1}} & =A_{n_{1}}^{(1)} \otimes I \otimes \cdots \otimes I, \quad A_{n_{1}+1}=J \otimes A_{1}^{(2)} \otimes I \otimes \cdots \otimes I, \ldots, \\
A_{n_{1}+n_{2}} & =J \otimes A_{n_{2}}^{(2)} \otimes I \otimes \cdots \otimes I, \ldots, \\
A_{n_{n_{-1}}+i_{0}} & =J \otimes \cdots \otimes J \otimes A_{i_{0}}^{(i)} \otimes I \otimes \cdots \otimes I\left(1 \leq i_{0} \leq n_{i}\right), \ldots, \\
A_{n_{t-1}+1} & =J \otimes \cdots \otimes J \otimes A_{1}^{(t)}, \ldots, \quad A_{n}=J \otimes \cdots \otimes J \otimes A_{n_{t}}^{(t)},
\end{aligned}
$$

where in $(17)$ there are $(i-1)$ factors of $J$. 
(f)

$$
\begin{aligned}
E_{0} & =E_{0}^{(1)} \otimes \cdots \otimes E_{0}^{(t-1)} \otimes E_{0}^{(t)}=|X|^{-1} J \\
E_{1} & =E_{0}^{(1)} \otimes \cdots \otimes E_{0}^{(t-1)} \otimes E_{1}^{(t)} \\
& \vdots \\
E_{n_{t}} & =E_{0}^{(1)} \otimes \cdots \otimes E_{0}^{(t-1)} \otimes E_{n_{t}}^{(t)} \\
E_{n_{t}+1} & =E_{0}^{(1)} \otimes \cdots \otimes E_{0}^{(t-2)} \otimes E_{1}^{(t-1)} \otimes I, \\
& \vdots \\
& =E_{0}^{(1)} \otimes \cdots \otimes E_{0}^{(t-2)} \otimes E_{n_{t-1}}^{(t-1)} \otimes I, \\
& \vdots \\
E_{n_{t}+n_{t-1}} & \\
E_{\overline{n_{i+1}}+i_{0}} & =E_{0}^{(1)} \otimes \cdots \otimes E_{0}^{(i-1)} \otimes E_{i_{0}}^{(i)} \otimes I \otimes \cdots \otimes I\left(1 \leq i_{0} \leq n_{i}\right) \\
& \vdots \\
E_{\overline{n_{2}}+1} & =E_{1}^{(1)} \otimes I \otimes \cdots \otimes I, \ldots, \quad E_{n}=E_{n_{1}}^{(1)} \otimes I \otimes \cdots \otimes I .
\end{aligned}
$$

For $i=1,2, \ldots, t$, let $P^{(i)}$ and $Q^{(i)}$ be respectively the $P$-matrix and the $Q$ matrix of $\mathfrak{X}^{(i)}$. For these, we put

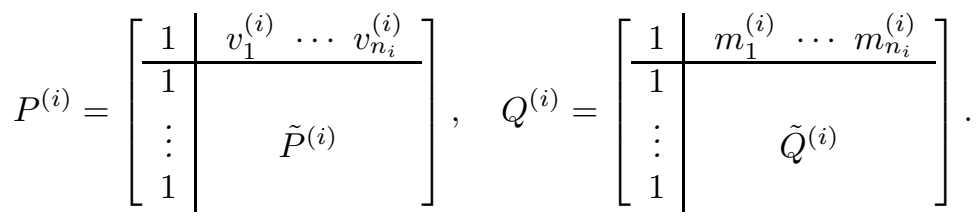

(g)

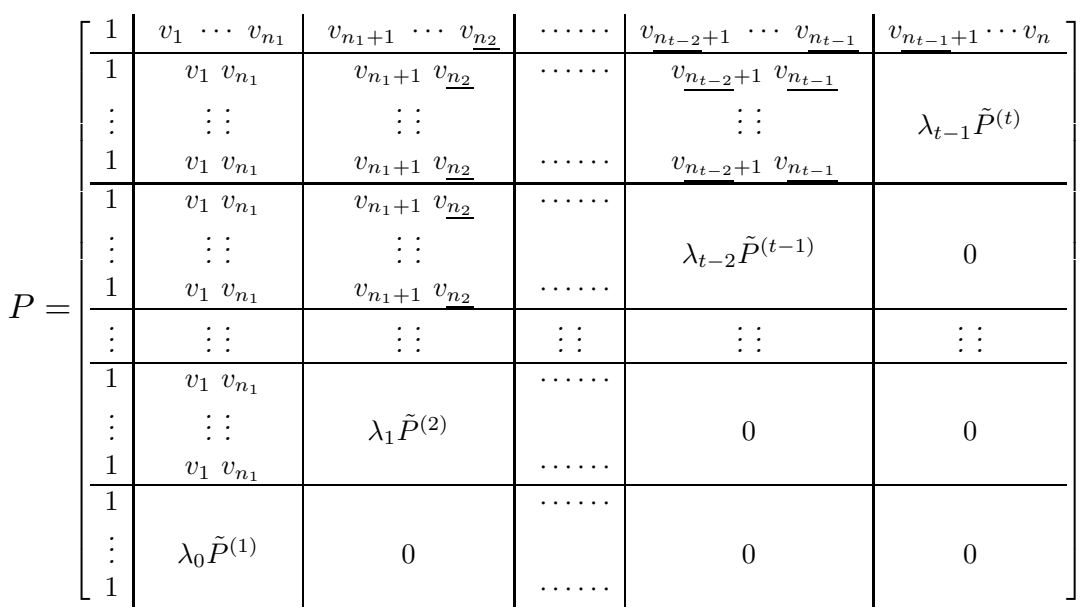


(h)

\begin{tabular}{|c|c|c|c|c|c|}
\hline 1 & $m_{1} \cdots m_{n_{t}}$ & $m_{n_{t}+1} \cdots m_{\overline{n_{t-1}}}$ & $\ldots \ldots$ & $m_{\overline{n_{3}}+1} \cdots m_{\overline{n_{2}}}$ & $m_{\overline{n_{2}}+1} \cdots m_{\overline{n_{1}}}$ \\
\hline 1 & $\begin{array}{c}m_{1} m_{n_{t}} \\
\quad \vdots \vdots \\
m_{1} m_{n_{t}}\end{array}$ & $\begin{array}{c}m_{n_{t}+1} m_{\overline{n_{t-1}}} \\
\vdots \vdots \\
m_{n_{t}+1} m_{\overline{n_{t-1}}}\end{array}$ & & $\begin{array}{c}m_{\overline{n_{3}}+1} m_{\overline{n_{2}}} \\
\vdots \vdots \\
m_{\overline{n_{3}}+1} m_{\overline{n_{2}}}\end{array}$ & $\mu_{2} \tilde{Q}^{(1)}$ \\
\hline 1 & $\begin{array}{c}m_{1} m_{n_{t}} \\
\vdots \vdots \\
m_{1} m_{n_{1}}\end{array}$ & $\begin{array}{c}m_{n_{t}+1} m_{\overline{n_{t-1}}} \\
\vdots \vdots \\
m_{n_{t}+1} m_{\overline{n_{t-1}}}\end{array}$ & & $\mu_{3} \tilde{Q}^{(2)}$ & 0 \\
\hline & $\vdots \vdots$ & $\vdots \vdots$ & $\vdots \vdots$ & $\vdots \vdots$ & $\vdots \vdots$ \\
\hline 1 & $\begin{array}{c}m_{1} m_{n_{t}} \\
\vdots \vdots \\
m_{1} m_{n_{t}}\end{array}$ & $\mu_{t} \tilde{Q}^{(t-1)}$ & $\ldots$ & 0 & 0 \\
\hline & $\mu_{t+1} \tilde{Q}^{(t)}$ & 0 & & 0 & 0 \\
\hline
\end{tabular}

Proof. (a) Let $\left(x_{1}, x_{2}, \ldots, x_{t}\right) \in X$. Then

$$
\begin{aligned}
v_{n_{i-1}}+i_{0} & =\left|\left\{\left(y_{1}, \ldots, y_{i}, x_{i+1}, \ldots, x_{t}\right) \in X \mid\left(x_{i}, y_{i}\right) \in R_{i_{0}}^{(i)}\right\}\right| \\
& =\left|X_{1}\right| \cdots\left|X_{i-1}\right| v_{i_{0}}^{(i)} .
\end{aligned}
$$

(b) Induction on $i$. For $i=1, \lambda_{1}=\sum_{j=0}^{n_{1}} v_{j}=\sum_{j=0}^{n_{1}} v_{j}^{(1)}=\left|X_{1}\right|$, by (a). Assume that it holds for $i(i \geq 1)$. Then, again by (a),

$$
\begin{aligned}
\lambda_{i+1} & =\lambda_{i}+\sum_{j=\underline{n_{i}}+1}^{n_{i+1}} v_{j} \\
& =\left|X_{1}\right| \cdots\left|X_{i}\right|+\left|X_{1}\right| \cdots\left|X_{i}\right| \sum_{i_{0}=1}^{n_{i+1}} v_{i_{0}}^{(i+1)} \\
& =\left|X_{1}\right| \cdots\left|X_{i}\right|+\left|X_{1}\right| \cdots\left|X_{i}\right|\left(\left|X_{i+1}\right|-1\right) \\
& =\left|X_{1}\right| \cdots\left|X_{i+1}\right| .
\end{aligned}
$$

(e), (f) These can be shown directly. Or these will follow by generalizing the results in Section 4 of [9] stated for the wreath product of two association schemes. Observe, however, that in what will follow we reversed the order of factors in tensor product expressions of adjacency matrices and irreducible idempotents.

(c) From (f),

$$
\begin{aligned}
m_{\overline{n_{i+1}}+i_{0}} & =\operatorname{tr} E_{\overline{n_{i+1}}+i_{0}} \\
& =\operatorname{tr} E_{0}^{(1)} \cdots \operatorname{tr} E_{0}^{(i-1)} \operatorname{tr} E_{i_{0}}^{(i)}\left|X_{i+1}\right| \cdots\left|X_{t}\right| \\
& =\left|X_{t}\right| \cdots\left|X_{i+1}\right| m_{i_{0}}^{(i)} .
\end{aligned}
$$

(d) Reverse induction on $i$, starting from $t$. 
(g) For the wreath product of two association schemes, this is also stated in Section 4 of [9]. The proof is left to the reader, as it can be shown just as in (h) below.

(h)

$$
\begin{aligned}
E_{\overline{n_{i+1}}+i_{0}}= & E_{0}^{(1)} \otimes \cdots \otimes E_{0}^{(i-1)} \otimes E_{i_{0}}^{(i)} \otimes I \otimes \cdots \otimes I \\
= & \left|X_{1}\right|^{-1} J \otimes \cdots \otimes\left|X_{i-1}\right|^{-1} J \otimes\left|X_{i}\right|^{-1} \sum_{j=0}^{n_{i}} q_{j i_{0}}^{(i)} A_{j}^{(i)} \otimes I \otimes \cdots \otimes I \\
= & |X|^{-1}\left\{\mu_{i+1} \sum_{j=1}^{n_{i}} q_{j i_{0}}^{(i)} J \otimes \cdots \otimes J \otimes A_{j}^{(i)} \otimes I \otimes \cdots \otimes I\right. \\
& \left.+m_{\overline{n_{i+1}}+i_{0}} J \otimes \cdots \otimes J \otimes I \otimes I \otimes \cdots \otimes I\right\} \\
= & |X|^{-1}\left\{\mu_{i+1} \sum_{j=1}^{n_{i}} q_{j i_{0}}^{(i)} A_{\underline{n_{i-1}}}+j+m_{\overline{n_{i+1}}+i_{0}}\left(A_{0}+A_{1}+\cdots+A_{\underline{n_{i-1}}}\right)\right\},
\end{aligned}
$$

where the last equality is obtained by replacing various $J$ by the corresponding sum of adjacency matrices for $\mathfrak{X}^{(j)}$ for $j=i-1, i-2, \ldots, 1$.

Remark 6. From the expression of $P$ in Theorem 5 (g), we have:

$$
\theta_{0}^{(i)}, \theta_{1}^{(i)}, \ldots, \theta_{n}^{(i)}=\underbrace{\theta_{0}^{(i)}, \theta_{0}^{(i)}, \ldots, \theta_{0}^{(i)}}_{\overline{n_{i+1}}+1}, \theta_{\overline{n_{i+1}}+1}^{(i)}, \ldots, \theta_{\overline{n_{i}}}^{(i)}, \underbrace{0, \ldots, 0}_{\underline{\underline{n_{i-1}}}} .
$$

Theorem 7. Let $\mathfrak{X}=\left(X=X_{1} \times \cdots \times X_{t},\left\{R_{j}\right\}_{j=0}^{n}\right)=\mathfrak{X}^{(1)} \imath \cdots \curlywedge \mathfrak{X}^{(t)}$ be a symmetric association scheme which is given as the wreath product of the symmetric association schemes $\mathfrak{X}^{(i)}=\left(X_{i},\left\{R_{j}^{(i)}\right\}_{j=0}^{n_{i}}\right)$.

(a) The first intersection matrix $L^{(i)}=\left(\underline{p}_{\underline{n_{i-1}}}^{k}+1, l\right)_{0 \leq k, l \leq n}$ of level $i$ is

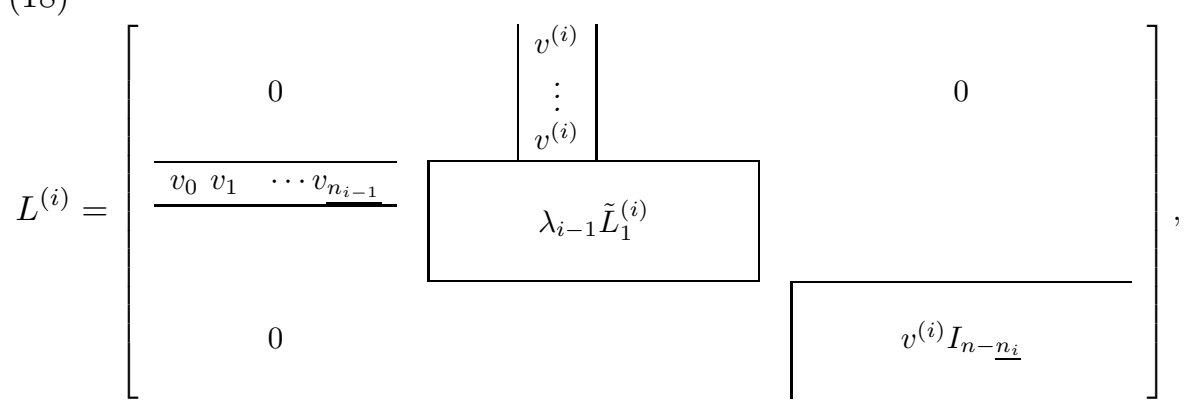

where the upper left diagonal block matrix in (18) is the zero matrix of size $\underline{n_{i-1}}+1$ (with rows and columns indexed by $0,1, \ldots, \underline{n_{i-1}}$ ), and ${\tilde{L_{1}}}^{\left({ }^{(i)}\right.}$ is the submatrix $\left(p_{1 l}^{(i) k}\right)_{1 \leq k, l \leq n_{i}}$ of the first intersection matrix $L_{1}^{(i)}=\left(p_{1 l}^{(i) k}\right)_{0 \leq k, l \leq n_{i}}$ of $\mathfrak{X}^{(i)}$. 


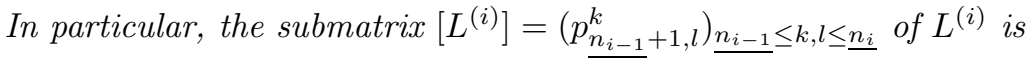

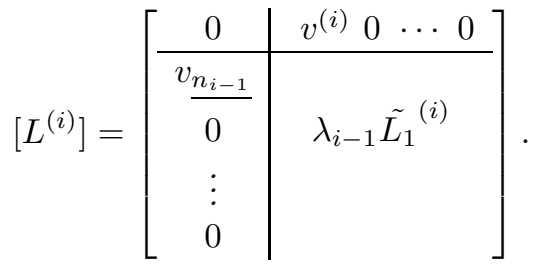

(b) The first dual intersection matrix $M^{(i)}=\left(q \frac{k}{n_{i+1}+1, l}\right)_{0 \leq k, l \leq n}$ of level $i$ is (20)

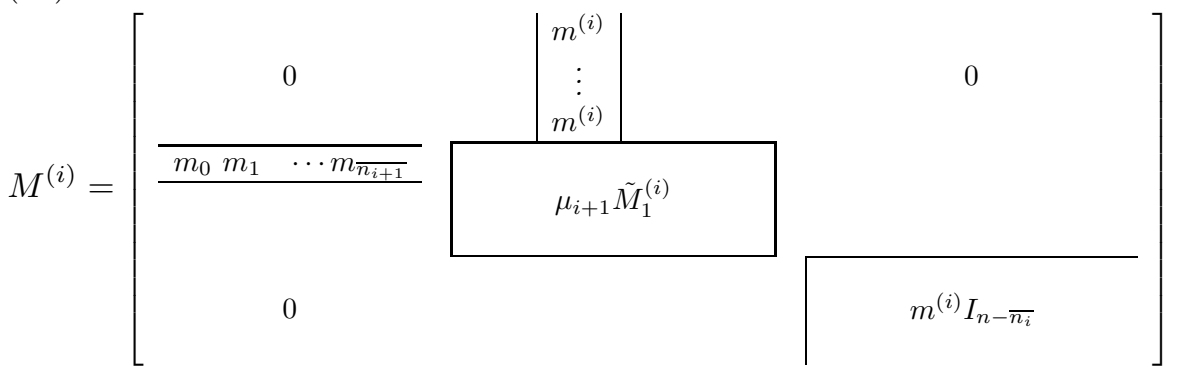

where the upper left diagonal block matrix in (20) is the zero matrix of size $\overline{n_{i+1}}+1$ (with rows and columns indexed by $0,1, \ldots, \overline{n_{i+1}}$ ), and $\tilde{M}_{1}^{(i)}$ is the submatrix $\left(q_{1 l}^{(i) k}\right)_{1 \leq k, l \leq n_{i}}$ of the first intersection matrix $M_{1}^{(i)}=\left(q_{1 l}^{(i) k}\right)_{0 \leq k, l \leq n_{i}}$ of $\mathfrak{X}^{(i)}$.

In particular, the submatrix $\left[M^{(i)}\right]=\left(q_{\overline{n_{i+1}}+1, l}^{k}\right) \overline{n_{i+1}} \leq k, l \leq \overline{n_{i}}$ of $M^{(i)}$ is

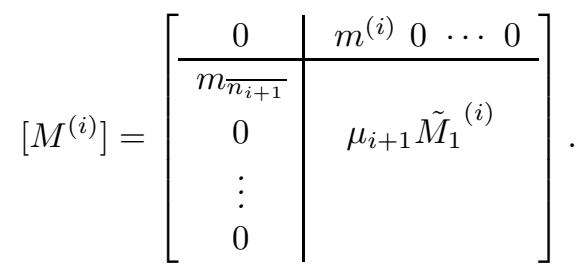

Proof. Here we will give only a proof for (b). From Theorem 5(f),

$$
\begin{aligned}
& E_{\overline{n_{i+1}}+1} \circ E_{\overline{n_{i+1}}+l} \\
= & \left(E_{0}^{(1)} \otimes \cdots \otimes E_{0}^{(i-1)} \otimes E_{1}^{(i)} \otimes I \otimes \cdots \otimes I\right) \\
& \circ\left(E_{0}^{(1)} \otimes \cdots \otimes E_{0}^{(i-1)} \otimes E_{l}^{(i)} \otimes I \otimes \cdots \otimes I\right) \\
= & \left(\left|X_{1}\right| \cdots\left|X_{i-1}\right|\right)^{-1} E_{0}^{(1)} \otimes \cdots \otimes E_{0}^{(i-1)} \otimes E_{1}^{(i)} \circ E_{l}^{(i)} \otimes I \otimes \cdots \otimes I \\
= & |X|^{-1}\left\{\sum_{k=1}^{n_{i}} \mu_{i+1} q_{1 l}^{(i) k} E_{0}^{(1)} \otimes \cdots \otimes E_{0}^{(i-1)} \otimes E_{k}^{(i)} \otimes I \otimes \cdots \otimes I\right. \\
& \left.+m^{(i)} \delta_{1 l} E_{0}^{(1)} \otimes \cdots \otimes E_{0}^{(i-1)} \otimes E_{0}^{(i)} \otimes I \otimes \cdots \otimes I\right\}
\end{aligned}
$$




$$
=|X|^{-1}\left\{\sum_{k=1}^{n_{i}} \mu_{i+1} q_{1 l}^{(i) k} E_{\overline{n_{i+1}}+k}+m^{(i)} \delta_{1 l}\left(E_{0}+\cdots+E_{\overline{n_{i+1}}}\right)\right\},
$$

where the last equality is obtained by replacing various $I$ by the corresponding sum of irreducible idempotents for $\mathfrak{X}^{(j)}$, for $j=i+1, \ldots, t$.

Let $j>i, 1 \leq l \leq n_{j}$, or let $j=t, l=0$. Then

$$
\begin{aligned}
& E_{\overline{n_{i+1}}+1} \circ E_{\overline{n_{j+1}}+l} \\
= & \left(E_{0}^{(1)} \otimes \cdots \otimes E_{0}^{(i-1)} \otimes E_{1}^{(i)} \otimes I \otimes \cdots \otimes I \otimes I \otimes I \otimes \cdots \otimes I\right)
\end{aligned}
$$$$
\circ\left(E_{0}^{(1)} \otimes \cdots \otimes E_{0}^{(i-1)} \otimes E_{0}^{(i)} \otimes E_{0}^{(i+1)} \otimes \cdots \otimes E_{0}^{(j-1)} \otimes E_{l}^{(j)} \otimes I \otimes \cdots \otimes I\right)
$$$$
=\left|X_{1}\right|^{-1} E_{0}^{(1)} \otimes \cdots \otimes\left|X_{i-1}\right|^{-1} E_{0}^{(i-1)} \otimes E_{1}^{(i)} \circ E_{0}^{(i)} \otimes\left|X_{i+1}\right|^{-1} I \otimes \cdots
$$$$
\otimes\left|X_{j-1}\right|^{-1} I \otimes I \circ E_{l}^{(j)} \otimes I \otimes \cdots \otimes I
$$

$$
=|X|^{-1} m_{\overline{n_{j+1}+l}} E_{\overline{n_{i+1}}+1} \text {, }
$$

where we note that

$$
E_{1}^{(i)} \circ E_{0}^{(i)}=\left|X_{i}\right|^{-1} E_{1}^{(i)}, I \circ E_{l}^{(j)}=\left|X_{j}\right|^{-1} m_{l}^{(j)} I .
$$

Similarly to the just above case, one shows, for $j<i$, that $E_{\overline{n_{i+1}}+1} \circ$ $E_{\overline{n_{j+1}}+l}=|X|^{-1} m^{(i)} E_{\overline{n_{j+1}}+l}$.

Remark 8. $\lambda_{i-1} L_{1}^{(i)}$ equals $\left[L^{(i)}\right]$ except for the $(2,1)$ entry, namely the $(2,1)$ entry of $\lambda_{i-1} L_{1}^{(i)}$ is $\lambda_{i-1}=\sum \frac{n_{i-1}}{j=0} v_{j}$, whereas that of $\left[L^{(i)}\right]$ is $v_{n_{i-1}}$ (cf. (19)). Also, $\mu_{i+1} M_{1}^{(i)}$ equals $\left[M^{(i)}\right]$ except for the $(2,1)$ entry, namely the $(2,1)$ entry of $\mu_{i+1} M_{1}^{(i)}$ is $\mu_{i+1}=\sum_{j=0}^{\overline{n_{i+1}}} m_{j}$, whereas that of $\left[M^{(i)}\right]$ is $m_{\overline{n_{i+1}}}$ (cf. (21)).

Proposition 9. Let $\mathfrak{X}=\left(X=X_{1} \times \cdots \times X_{t},\left\{R_{j}\right\}_{j=0}^{n}\right)=\mathfrak{X}^{(1)} \prec \cdots \curlywedge \mathfrak{X}^{(t)}$ be a symmetric association scheme which is given as the wreath product of the symmetric association schemes $\mathfrak{X}^{(i)}=\left(X_{i},\left\{R_{j}^{(i)}\right\}_{j=0}^{n_{i}}\right)$. Then we have:

(a) $\theta_{0}^{(i)}, \ldots, \theta_{n}^{(i)}$ are real numbers and eigenvalues of $L^{(i)}$ for $i=1,2, \ldots, t$. Further, $\left|\theta_{j}^{(i)}\right| \leq v^{(i)}$ for $i=1,2, \ldots, t, 0 \leq j \leq n$.

If in addition $\mathfrak{X}$ is a weak metric scheme, then we have:

(b)

$$
\begin{aligned}
\psi_{0}^{(1)}(x)= & 1, \psi_{1}^{(1)}(x)=x, \\
c_{i_{0}}^{(1)} \psi_{i_{0}}^{(1)}(x)= & \left(x-a_{i_{0}-1}^{(1)}\right) \psi_{i_{0}-1}^{(1)}(x)-b_{i_{0}-2}^{(1)} \psi_{i_{0}-2}^{(1)}(x)\left(2 \leq i_{0} \leq n_{1}\right) \\
\alpha_{0}^{(1)}= & \alpha_{1}^{(1)}=\cdots=\alpha_{n_{1}}^{(1)}=0 \text { for } 2 \leq i \leq t, \\
\psi_{1}^{(i)}(x)= & x, \psi_{2}^{(i)}(x)=c_{2}^{(i)-1}\left(x^{2}-a_{1}^{(i)} x\right), \\
c_{i_{0}}^{(i)} \psi_{i_{0}}^{(i)}(x)= & \left(x-a_{i_{0}-1}^{(i)}\right) \psi_{i_{0}-1}^{(i)}(x)-b_{i_{0}-2}^{(i)} \psi_{i_{0}-2}^{(i)}(x) \\
& +\alpha_{i_{0}-1}^{(i)}\left(v_{0}+\cdots+v_{n_{i-1}}\right) x\left(3 \leq i_{0} \leq n_{i}\right),
\end{aligned}
$$




$$
\begin{aligned}
\alpha_{1}^{(i)} & =0, \alpha_{2}^{(i)}=-c_{2}^{(i)^{-1}} v^{(i)}=-c_{2}^{(i)^{-1}} b_{0}^{(i)}, \\
c_{i_{0}}^{(i)} \alpha_{i_{0}}^{(i)} & =-\left(b_{i_{0}-2}^{(i)} \alpha_{i_{0}-2}^{(i)}+a_{i_{0}-1}^{(i)} \alpha_{i_{0}-1}^{(i)}\right)\left(3 \leq i_{0} \leq n_{i}\right) .
\end{aligned}
$$

Proof. (a) The first statement follows from $P L^{(i)} P^{-1}=\operatorname{diag}\left(\theta_{0}^{(i)}, \ldots, \theta_{n}^{(i)}\right)$ and the second one does from Perron-Frobenius' theorem.

(b) This follows from the proof (b) $\Rightarrow$ (c) of Theorem 2.

Lemma 10. Let $\mathfrak{X}=\left(X=X_{1} \times \cdots \times X_{t},\left\{R_{j}\right\}_{j=0}^{n}\right)=\mathfrak{X}^{(1)} \prec \cdots \curlywedge \mathfrak{X}^{(t)}$ be a weak metric scheme. Then, for $2 \leq i \leq t$,

$$
\left(1+\alpha_{2}^{(i)}+\cdots+\alpha_{n_{i}}^{(i)}\right) \lambda_{i-1}=\frac{(-1)^{n_{i}} \theta \frac{(i)}{n_{i+1}+1} \cdots \theta \frac{(i)}{n_{i}}}{c_{2}^{(i)} c_{3}^{(i)} \cdots c_{n_{i}}^{(i)}} .
$$

Proof. From (24) and in view of Remark 8, with $\alpha_{0}^{(i)}=1$ we have:

$$
\left[\begin{array}{cccccc}
1 & & & & & \\
\tilde{b}_{0}^{(i)} & \tilde{c}_{2}^{(i)} & & & & \\
& \tilde{a}_{2}^{(i)} & \tilde{c}_{3}^{(i)} & & & \\
& \tilde{b}_{2}^{(i)} & \tilde{a}_{3}^{(i)} & \tilde{c}_{4}^{(i)} & & \\
& & \ddots & \ddots & \ddots & \\
& & & \tilde{b}_{n_{i}-2}^{(i)} & \tilde{a}_{n_{i}-1}^{(i)} & \tilde{c}_{n_{i}}^{(i)}
\end{array}\right]\left[\begin{array}{c}
\alpha_{0}^{(i)} \\
\alpha_{2}^{(i)} \\
\alpha_{3}^{(i)} \\
\vdots \\
\vdots \\
\alpha_{n_{i}}^{(i)}
\end{array}\right]=\left[\begin{array}{c}
1 \\
0 \\
0 \\
\vdots \\
\vdots \\
0
\end{array}\right]
$$

if the first intersection matrix $L_{1}^{(i)}$ of $\mathfrak{X}^{(i)}$ is given by

$$
\left[\begin{array}{cccccc}
\tilde{a}_{0}^{(i)} & \tilde{b}_{0}^{(i)} & & & & \\
\tilde{c}_{1}^{(i)} & \tilde{a}_{1}^{(i)} & \tilde{b}_{1}^{(i)} & & & \\
& \tilde{c}_{2}^{(i)} & \tilde{a}_{2}^{(i)} & \tilde{b}_{2}^{(i)} & & \\
& & \ddots & \ddots & \ddots & \\
& & & \tilde{c}_{n_{i}-1}^{(i)} & \tilde{a}_{n_{i}-1}^{(i)} & \tilde{b}_{n_{i}-1}^{(i)} \\
& & & & \tilde{c}_{n_{i}}^{(i)} & \tilde{a}_{n_{i}}^{(i)}
\end{array}\right] .
$$

By Remark 8 and the shape of $P$ (cf. Theorem $5(\mathrm{~g})$ ), (25) is equivalent to:

$$
\left(1+\alpha_{2}^{(i)}+\cdots+\alpha_{n_{i}}^{(i)}\right)=\frac{(-1)^{n_{i}} p_{11}^{(i)} \cdots p_{n_{i} 1}^{(i)}}{\tilde{c}_{2}^{(i)} \tilde{c}_{3}^{(i)} \cdots \tilde{c}_{n_{i}}^{(i)}},
$$

with $P^{(i)}=\left(p_{k l}^{(i)}\right)_{0 \leq k, l \leq n_{i}}$ the $P$-matrix of $\mathfrak{X}^{(i)}$.

It can be shown (cf. [2, Theorem 1.3, p. 197]) that, if we define

$$
\begin{aligned}
\tilde{c}_{j}^{(i)} P_{j}(x) & =\left(x-\tilde{a}_{j-1}^{(i)}\right) P_{j-1}(x)-\tilde{b}_{j-2}^{(i)} P_{j-2}(x)\left(2 \leq j \leq n_{i}\right), \\
P_{0}(x) & =1, P_{1}(x)=x,
\end{aligned}
$$

and

$$
P_{n_{i}+1}(x)=\left(x-\tilde{a}_{n_{i}}^{(i)}\right) P_{n_{i}}(x)-\tilde{b}_{n_{i}-1}^{(i)} P_{n_{i}-1}(x),
$$


then

$$
\begin{aligned}
P_{n_{i}+1}(x) & =\frac{1}{\tilde{c}_{2}^{(i)} \tilde{c}_{3}^{(i)} \cdots \tilde{c}_{n_{i}}^{(i)}}\left(x-p_{01}^{(i)}\right)\left(x-p_{11}^{(i)}\right) \cdots\left(x-p_{n_{i} 1}^{(i)}\right) \\
& =\left(x-p_{01}^{(i)}\right) \sum_{j=0}^{n_{i}} P_{j}(x) .
\end{aligned}
$$

So we see that $\sum_{j=0}^{n_{i}} P_{j}(0)=\operatorname{RHS}$ of $(27)$, and (26) is satisfied with $\alpha_{0}^{(i)}$, $\alpha_{2}^{(i)}, \ldots, \alpha_{n_{i}}^{(i)}$ respectively replaced by $P_{0}(0), P_{2}(0), \ldots, P_{n_{i}}(0)$. As $\mathfrak{X}^{(i)}$ is a metric scheme, the square matrix on the LHS of (26) is invertible, and hence $\alpha_{0}^{(i)}=P_{0}(0), \alpha_{2}^{(i)}=P_{2}(0), \ldots, \alpha_{n_{i}}^{(i)}=P_{n_{i}}(0)$. In particular, $\sum_{j=0}^{n_{i}} P_{j}(0)=$ $\alpha_{0}^{(i)}+\alpha_{2}^{(i)}+\cdots+\alpha_{n_{i}}^{(i)}$.

Theorem 11. Let $\mathfrak{X}=\left(X=X_{1} \times \cdots \times X_{t},\left\{R_{j}\right\}_{j=0}^{n}\right)=\mathfrak{X}^{(1)} \prec \cdots \prec \mathfrak{X}^{(t)}$ be a weak metric scheme. Assume $\psi^{(i)}(x)$ 's are as in $(22)$ and $(23)$. Let $\psi_{n_{1}+1}^{(1)}(x)$ be the polynomial of degree $n_{1}+1$ defined by:

$$
\psi_{n_{1}+1}^{(1)}(x)=\left(x-a_{n_{1}}^{(1)}\right) \psi_{n_{1}}^{(1)}(x)-b_{n_{1}-1}^{(1)} \psi_{n_{1}-1}^{(1)}(x) .
$$

Then

(28) $\psi_{n_{1}+1}^{(1)}(x)=\frac{1}{c_{2}^{(1)} \cdots c_{n_{1}}^{(1)}}\left(x-\theta_{0}^{(1)}\right)\left(x-\theta_{\frac{1}{n_{2}}+1}^{(1)}\right)\left(x-\theta_{\overline{n_{2}}+2}^{(1)}\right) \cdots\left(x-\theta_{\overline{n_{1}}}^{(1)}\right)$

$$
=\left(x-\theta_{0}^{(1)}\right) \sum_{j=0}^{n_{1}} \psi_{j}^{(1)}(x) \text {. }
$$

For $2 \leq i \leq t$, let $\psi_{n_{i}+1}^{(i)}(x)$ be the polynomial of degree $n_{i}+1$ defined by:

$$
\psi_{n_{i}+1}^{(i)}(x)=\left(x-a_{n_{i}}^{(i)}\right) \psi_{n_{i}}^{(i)}(x)-b_{n_{i}-1}^{(i)} \psi_{n_{i}-1}^{(i)}(x)+\alpha_{n_{i}}^{(i)}\left(v_{0}+\cdots+v_{\underline{n_{i-1}}}\right) x+\tau_{i},
$$

with

$$
\tau_{i}=\frac{(-1)^{n_{i}+1} \theta_{0}^{(i)} \theta_{\overline{n_{i+1}}+1}^{(i)} \cdots \theta_{\overline{n_{i}}}^{(i)}}{c_{2}^{(i)} \cdots c_{n_{i}}^{(i)}}
$$

Then

$$
\begin{aligned}
\psi_{n_{i}+1}^{(i)}(x) & =\frac{1}{c_{2}^{(i)} \cdots c_{n_{i}}^{(i)}}\left(x-\theta_{0}^{(i)}\right)\left(x-\theta_{n_{i+1}+1}^{(i)}\right) \cdots\left(x-\theta_{\overline{n_{i}}}^{(i)}\right) \\
& =\left(x-\theta_{0}^{(i)}\right) \sum_{j=0}^{n_{i}} \psi_{j}^{(i)}(x)
\end{aligned}
$$

with

$$
\psi_{0}^{(i)}(x)=\frac{(-1)^{n_{i}} \theta_{\overline{n_{i+1}}+1}^{(i)} \cdots \theta_{\overline{n_{i}}}^{(i)}}{c_{2}^{(i)} c_{3}^{(i)} \cdots c_{n_{i}}^{(i)}}
$$


Note here that

$$
\tau_{i}=-\theta_{0}^{(i)} \psi_{0}^{(i)}(x)=-v^{(i)} \psi_{0}^{(i)}(x)
$$

Proof. As $\psi_{k}^{(i)}(0)=0$ for $2 \leq i \leq t, 1 \leq k \leq n_{i}, \psi_{0}^{(i)}(x)$ is the constant polynomial in (33) if (31) and (32) are true.

Let $i=1$. From (22), we see that

$$
B\left[\begin{array}{c}
\psi_{0}^{(1)}(x) \\
\psi_{1}^{(1)}(x) \\
\vdots \\
\psi_{n_{1}}^{(1)}(x)
\end{array}\right]=\left[\begin{array}{c}
0 \\
\vdots \\
0 \\
\psi_{n_{1}+1}^{(1)}(x)
\end{array}\right]
$$

where $B=x I_{n_{1}+1}-{ }^{t}\left[L^{(1)}\right]$. So, by multiplying the adjoint $\operatorname{Adj}(B)$ on both sides, we get:

$$
|B|\left[\begin{array}{c}
\psi_{0}^{(1)}(x) \\
\psi_{1}^{(1)}(x) \\
\vdots \\
\psi_{n_{1}}^{(1)}(x)
\end{array}\right]=\operatorname{Adj}(B)\left[\begin{array}{c}
0 \\
\vdots \\
0 \\
\psi_{n_{1}+1}^{(1)}(x)
\end{array}\right]
$$

By looking at the first component, from (36) we have:

$$
|B|=c_{2}^{(1)} \cdots c_{n_{1}}^{(1)} \psi_{n_{1}+1}^{(1)}(x) .
$$

So

$$
\psi_{n_{1}+1}^{(1)}(x)=\frac{1}{c_{2}^{(1)} \cdots c_{n_{1}}^{(1)}} \operatorname{char} \operatorname{poly}\left(\left[L^{(1)}\right]\right),
$$

where char poly $\left(\left[L^{(1)}\right]\right)$ is an abbreviation for the characteristic polynomial of $\left[L^{(1)}\right]$. On the other hand, from $P L^{(1)} P^{-1}=\operatorname{diag}\left(\theta_{0}^{(1)}, \theta_{1}^{(1)}, \ldots, \theta_{n}^{(1)}\right)$ and (18), we have:

$$
\begin{aligned}
& \operatorname{char} \operatorname{poly}\left(\left[L^{(1)}\right]\right)\left(x-\theta_{0}^{(1)}\right)^{n-n_{1}} \\
= & \operatorname{char} \operatorname{poly}\left(L^{(1)}\right) \\
= & \left(x-\theta_{0}^{(1)}\right) \cdots\left(x-\theta_{n}^{(1)}\right) .
\end{aligned}
$$

In view of Remark 6 , this implies that

$$
\operatorname{char} \operatorname{poly}\left(\left[L^{(1)}\right]\right)=\left(x-\theta_{0}^{(1)}\right)\left(x-\theta_{\overline{n_{2}}+1}^{(1)}\right) \cdots\left(x-\theta_{\overline{n_{1}}}^{(1)}\right) .
$$

Now, (28) follows from (37) and (38). Also, we have (29) by multiplying both sides of (35) by $[1 \cdots 1]$ on the left (cf. (15)). 
Let $i \geq 2$. From (23), (24), and (30), we see that

$$
C\left[\begin{array}{c}
\psi_{0}^{(i)}(x)+\lambda_{i-1} \\
\psi_{1}^{(i)}(x) \\
\psi_{2}^{(i)}(x)+\alpha_{2}^{(i)} \lambda_{i-1} \\
\vdots \\
\vdots \\
\psi_{n_{i}}^{(i)}(x)+\alpha_{n_{i}}^{(i)} \lambda_{i-1}
\end{array}\right]=\left[\begin{array}{c}
x \psi_{0}^{(i)}(x) \\
\tau_{i} \\
0 \\
\vdots \\
0 \\
\triangle(x)
\end{array}\right]
$$

where $\triangle(x)=\psi_{n_{i}+1}^{(i)}(x)-\tau_{i}-\left(b_{n_{i}-1}^{(i)} \alpha_{n_{i}-1}^{(i)}+a_{n_{i}}^{(i)} \alpha_{n_{i}}^{(i)}\right) \lambda_{i-1}$, and $C=x I_{n_{i}+1}-$ ${ }^{t}\left(\lambda_{i-1} L_{1}^{(i)}\right)$ (cf. (12), Remark 8), with

$$
{ }^{t}\left(\lambda_{i-1} L_{1}^{(i)}\right)=\left[\begin{array}{cccccc}
0 & \lambda_{i-1} & & & & \\
v^{(i)} & a_{1}^{(i)} & c_{2}^{(i)} & & & \\
& b_{1}^{(i)} & a_{2}^{(i)} & c_{3}^{(i)} & & \\
& & \ddots & \ddots & \ddots & \\
& & & b_{n_{i}-2}^{(i)} & a_{n_{i}-1}^{(i)} & c_{n_{i}}^{(i)} \\
& & & & b_{n_{i}-1}^{(i)} & a_{n_{i}}^{(i)}
\end{array}\right]
$$

Putting $x=0$ in (39) and multiplying both sides on the left by $[1 \cdots 1]$, we get:

$$
\begin{aligned}
& -v^{(i)}\left(\psi_{0}^{(i)}(x)+\left(1+\alpha_{2}^{(i)}+\cdots+\alpha_{n_{i}}^{(i)}\right) \lambda_{i-1}\right) \\
= & \tau_{i}-\left(b_{n_{i}-1}^{(i)} \alpha_{n_{i}-1}^{(i)}+a_{n_{i}}^{(i)} \alpha_{n_{i}}^{(i)}\right) \lambda_{i-1} \\
\Leftrightarrow \tau_{i}= & -\left(b_{n_{i}-1}^{(i)} \alpha_{n_{i}-1}^{(i)}+a_{n_{i}}^{(i)} \alpha_{n_{i}}^{(i)}\right) \lambda_{i-1}
\end{aligned}
$$

(cf. (16), (25), (30), (33), (34)). So $\triangle(x)=\psi_{n_{i}+1}^{(i)}(x)$. Taking this into consideration, we have (32) after multiplying both sides of (39) on the left by [1 $\cdots 1]$.

On the other hand,

$$
\begin{aligned}
& \left(x-\theta_{0}^{(i)}\right)\left(x-\theta_{1}^{(i)}\right) \cdots\left(x-\theta_{n}^{(i)}\right) \\
= & \operatorname{det}\left(x I_{n+1}-{ }^{t} L^{(i)}\right) \\
= & \operatorname{det} D \\
= & x \frac{n_{i-1}}{}\left(x-\theta_{0}^{(i)}\right)^{\overline{n_{i+1}}} \operatorname{char} \operatorname{poly}\left(\lambda_{i-1} L_{1}^{(i)}\right),
\end{aligned}
$$


where

$$
D=\left[\begin{array}{c|c|c}
x I_{\underline{n_{i-1}}} & * & 0 \\
\hline 0 & x I_{n_{i}+1}-{ }^{t}\left(\lambda_{i-1} L^{(i)}\right) & 0 \\
\hline 0 & 0 & \left(x-v^{(i)}\right) I_{\overline{n_{i+1}}}
\end{array}\right] .
$$

$D$ can be obtained from $x I_{n+1}-{ }^{t} L^{(i)}$, by first adding the column $\left(n_{i-1}+1\right)$ to each of the columns one through $n_{i-1}$ and then adding the columns one through $\underline{n_{i-1}}$ to the column $\left(\underline{n_{i-1}}+1\right)$.

So, by Remark 6 ,

$$
\operatorname{char} \operatorname{poly}\left(\lambda_{i-1} L_{1}^{(i)}\right)=\left(x-\theta_{0}^{(i)}\right)\left(x-\theta_{\overline{n_{i+1}}+1}^{(i)}\right) \cdots\left(x-\theta_{\overline{n_{i}}}^{(i)}\right) .
$$

Now, after multiplying both sides of $(39)$ by $\operatorname{Adj}(C)$ on the left and looking at the second component, we get:

$$
x \operatorname{char} \operatorname{poly}\left(\lambda_{i-1} L_{1}^{(i)}\right)=\left|C^{\prime}\right|,
$$

where $C^{\prime}$ is the matrix obtained from $C$ by replacing the second column by

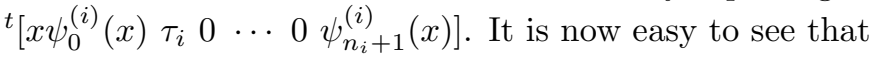

$$
\left|C^{\prime}\right|=\left(c_{2}^{(i)} \cdots c_{n_{i}}^{(i)} \psi_{n_{i}+1}^{(i)}(x)\right) x .
$$

Thus char $\operatorname{poly}\left(\lambda_{i-1} L_{1}^{(i)}\right)=c_{2}^{(i)} \cdots c_{n_{i}}^{(i)} \psi_{n_{i}+1}^{(i)}(x)$. This shows (31).

\section{Weak cometric schemes}

Let $\mathfrak{X}=\left(X=X_{1} \times \cdots \times X_{t},\left\{R_{j}\right\}_{j=0}^{n}\right)=\mathfrak{X}^{(1)} \prec \cdots \prec \mathfrak{X}^{(t)}$ be the symmetric association scheme which is given as the wreath product of the symmetric association schemes $\mathfrak{X}^{(i)}=\left(X_{i},\left\{R_{j}^{(i)}\right\}_{j=0}^{n_{i}}\right)$ (cf. Section 2 ). Then such a symmetric association scheme $\mathfrak{X}$ will be called a weak cometric scheme if it satisfies the equivalent conditions in Theorem 13.

Lemma 12. Let $W_{1}, \ldots, W_{m}, W_{1}^{\prime}, \ldots, W_{n}^{\prime}\left(m, n \in \mathbb{Z}_{\geq 0}\right), S, T$ be matrices with entries in a field. Assume that $W_{1}, \ldots, W_{m}, W_{1}^{\prime}, \ldots, W_{n}^{\prime}$ are nonzero. If $W_{1} \otimes \cdots \otimes W_{m} \otimes S \otimes W_{1}^{\prime} \otimes \cdots \otimes W_{n}^{\prime}=W_{1} \otimes \cdots \otimes W_{m} \otimes T \otimes W_{1}^{\prime} \otimes \cdots \otimes W_{n}^{\prime}$, then we have $S=T$.

Proof. For this, we only need to show:

$W \otimes S=W \otimes T$ and $W \neq 0$, or $S \otimes W=T \otimes W$ and $W \neq 0 \Rightarrow S=T$.

But this is trivial to see. 
Theorem 13. The following are equivalent.

(a)

(i) The submatrix $\left[M^{(i)}\right]=\left(q \frac{k}{n_{i+1}+1}, l\right) \overline{n_{i+1}} \leq k, l \leq \overline{n_{i}}$ of

$$
M^{(i)}=\left(q \frac{k}{n_{i+1}+1, l}\right)_{0 \leq k, l \leq n}
$$

is a tridiagonal matrix with nonzero off-diagonal entries for $i=1,2$, $\ldots, t$.

Moreover, $M^{(i)}$ has the following entries.

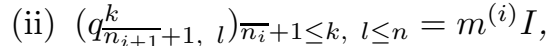

(iii) $q \frac{k}{n_{i+1}+1}, \overline{n_{i+1}}+1=m^{(i)}$ for $0 \leq k \leq \overline{n_{i+1}}$,

(iv) $q_{\overline{n_{i+1}}+1, l}^{\overline{n_{i+1}}+1}=m_{l}$ for $0 \leq l \leq \overline{n_{i+1}}$,

(v) all the other entries not appearing in (i)-(iv) are zeros.

(b) There are polynomials with real coefficients $\phi_{0}^{(t)}(x)$ of degree $0\left(\phi_{0}^{(t)}(x)=\right.$ $\left.|X|^{-1}\right), \phi_{k}^{(i)}(x)$ of degree $k$ for $i=1, \ldots, t, 1 \leq k \leq n_{i}$, with $\phi_{k}^{(i)}(0)=0$, $(1 \leq i \leq t-1)$, and real numbers $\beta_{0}^{(t)}=\cdots=\beta_{n_{t}}^{(t)}=0, \beta_{1}^{(t-1)}, \ldots, \beta_{n_{t-1}}^{(t-1)}, \ldots$, $\beta_{1}^{(1)}, \ldots, \beta_{n_{1}}^{(1)}$ such that

$$
E_{\overline{n_{i+1}}+i_{0}}=\phi_{i_{0}}^{(i)}\left(E_{\overline{n_{i+1}}+1}\right)+\beta_{i_{0}}^{(i)}\left(E_{0}+\cdots+E_{\overline{n_{i+1}}}\right)
$$

for $i=1,2 \ldots, t, 1 \leq i_{0} \leq n_{i}$, or $i=t, i_{0}=0$.

(c) q-numbers satisfy

$$
q_{j, \overline{n_{i+1}}+i_{0}}=\tilde{\phi}_{i_{0}}^{(i)}\left(\omega_{j}^{(i)}\right)+\beta_{i_{0}}^{(i)}\left(q_{j 0}+\cdots+q_{j}, \overline{n_{i+1}}\right)
$$

for $i=1, \ldots, t, 1 \leq i_{0} \leq n_{i}$, or $i=t, i_{0}=0, j=0,1, \ldots, n$.

Here $\beta_{i_{0}}^{(i)}$ 's are the same as in $(\mathrm{b}), \tilde{\phi}_{i_{0}}^{(i)}(x)=|X| \phi_{i_{0}}^{(i)}\left(|X|^{-1} x\right)$, and $\omega_{j}^{(i)}=$ $q_{j}, \overline{n_{i+1}}+1$.

(d) $\mathfrak{X}^{(i)}=\left(X_{i},\left\{R_{j}^{(i)}\right\}_{j=0}^{n_{i}}\right)$ is a cometric scheme for $i=1, \ldots, t$.

Proof. First, we note the following: for $i=1, \ldots, t, 1 \leq i_{0} \leq n_{i}$, or $i=t, i_{0}=$ 0 ,

$$
E_{\overline{n_{i+1}}+i_{0}} \circ E_{l}=|X|^{-1} m_{l} E_{\overline{n_{i+1}}+i_{0}}\left(0 \leq l \leq \overline{n_{i+1}}\right) .
$$

For this, we need to see: for $t \geq j>i, 1 \leq k \leq n_{j}$, or $j=t, k=0$,

$$
E_{\overline{n_{i+1}}+i_{0}} \circ E_{\overline{n_{j+1}}+k}=|X|^{-1} m_{\overline{n_{j+1}}+k} E_{\overline{n_{i+1}}+i_{0}} \text {. }
$$

But this can be shown just as in the proof of Theorem 7 .

(a) $\Rightarrow$ (b) Assume that $\left[M^{(i)}\right]=\left(q \frac{k}{n_{i+1}}, l\right) \overline{n_{i+1}} \leq k, l \leq \overline{n_{i}}$ is given by:

$$
\left[M^{(i)}\right]=\left[\begin{array}{ccccc}
e_{0}^{(i)} & f_{0}^{(i)} & & & \\
g_{1}^{(i)} & e_{1}^{(i)} & f_{1}^{(i)} & & \\
& \ddots & \ddots & \ddots & \\
& & g_{n_{i}-1}^{(i)} & e_{n_{i}-1}^{(i)} & f_{n_{i}-1}^{(i)} \\
& & & g_{n_{i}}^{(i)} & e_{n_{i}}^{(i)}
\end{array}\right]
$$


Observe here that $e_{0}^{(i)}=0, f_{0}^{(i)}=m^{(i)}, g_{1}^{(i)}=m \overline{n_{i+1}}$.

Define the polynomials $\phi_{i}^{(t)}(x)$ of degree $i\left(0 \leq i \leq n_{t}\right)$ recursively by:

$$
\begin{gathered}
\phi_{0}^{(t)}(x)=|X|^{-1}, \phi_{1}^{(t)}(x)=x, \\
x \phi_{i}^{(t)}(x)=f_{i-1}^{(t)} \phi_{i-1}^{(t)}(x)+e_{i}^{(t)} \phi_{i}^{(t)}(x)+g_{i+1}^{(t)} \phi_{i+1}^{(t)}(x)(i \geq 1) .
\end{gathered}
$$

Also, define $\beta_{0}^{(t)}=\beta_{1}^{(t)}=\cdots=\beta_{n_{t}}^{(t)}=0$. Then

$$
E_{i_{0}}=\phi_{i_{0}}^{(t)}\left(E_{1}\right)+\beta_{i_{0}}^{(t)} E_{0} \text { for } 0 \leq i_{0} \leq n_{t} .
$$

Here the multiplication is the Hadamard product and we understand that $\phi_{0}^{(t)}\left(E_{1}\right)=|X|^{-1} J=E_{0}$.

Starting from $t-1$, we apply an inductive argument to show (40) for each $i=t-1, \ldots, 1,1 \leq i_{0} \leq n_{i}$.

Before proceeding further, we note, using (42), that (a) is equivalent to:

$$
E_{\overline{n_{i+1}}+1} \circ E_{l}=|X|^{-1} m_{l} E_{\overline{n_{i+1}}+1}\left(0 \leq l \leq \overline{n_{i+1}}\right),
$$

$E_{\overline{n_{i+1}}+1} \circ E_{\overline{n_{i+1}}+1}=|X|^{-1}\left\{m^{(i)}\left(E_{0}+\cdots+E_{\overline{n_{i+1}}}\right)+e_{1}^{(i)} E_{\overline{n_{i+1}}+1}+g_{2}^{(i)} E_{\overline{n_{i+1}}+2}\right\}$

with $g_{2}^{(i)} \neq 0$,

$$
\begin{aligned}
& E_{\overline{n_{i+1}}+1} \circ E_{\overline{n_{i+1}}+i_{0}} \\
= & |X|^{-1}\left\{f_{i_{0}-1}^{(i)} E_{\overline{n_{i+1}}+i_{0}-1}+e_{i_{0}}^{(i)} E_{\overline{n_{i+1}}+i_{0}}+g_{i_{0}+1}^{(i)} E_{\overline{n_{i+1}}+i_{0}+1}\right\}\left(2 \leq i_{0} \leq n_{i}\right)
\end{aligned}
$$

with $f_{i_{0}-1}^{(i)} \neq 0\left(2 \leq i_{0} \leq n_{i}\right), g_{i_{0}+1}^{(i)} \neq 0\left(2 \leq i_{0} \leq n_{i}-1\right), g_{n_{i}+1}^{(i)}=0$,

$$
E_{\overline{n_{i+1}}+1} \circ E_{l}=|X|^{-1} m^{(i)} E_{l}\left(\bar{n}_{i}+1 \leq l \leq n\right) .
$$

Define $\phi_{1}^{(i)}(x)=x, \beta_{1}^{(i)}=0$. Then

$$
E_{\overline{n_{i+1}}+1}=\phi_{1}^{(i)}\left(E_{\overline{n_{i+1}}+1}\right)+\beta_{1}^{(i)}\left(E_{0}+\cdots+E_{\overline{n_{i+1}}}\right) .
$$

Also, if we define

$$
\phi_{2}^{(i)}(x)=g_{2}^{(i)^{-1}}\left(|X| x^{2}-e_{1}^{(i)} x\right), \beta_{2}^{(i)}=-g_{2}^{(i)^{-1}} m^{(i)},
$$

then

$$
E_{\overline{n_{i+1}}+2}=\phi_{2}^{(i)}\left(E_{\overline{n_{i+1}}+1}\right)+\beta_{2}^{(i)}\left(E_{0}+\cdots+E_{\overline{n_{i+1}}}\right) .
$$

Assume that $\phi_{1}^{(i)}, \ldots, \phi_{i_{0}}^{(i)}, \beta_{1}^{(i)}, \ldots, \beta_{i_{0}}^{(i)}\left(2 \leq i_{0}<n_{i}\right)$ are defined so that

$$
E_{\overline{n_{i+1}}+j_{0}}=\phi_{j_{0}}^{(i)}\left(E_{\overline{n_{i+1}}+1}\right)+\beta_{j_{0}}^{(i)}\left(E_{0}+\cdots+E_{\overline{n_{i+1}}}\right),
$$

and $\phi_{j_{0}}^{(i)}(0)=0$ holds for all $1 \leq j_{0} \leq i_{0}$.

Define the polynomial $\phi_{i_{0}+1}^{(i)}(x)$ and $\beta_{i_{0}+1}^{(i)}$ respectively by:

$$
\begin{aligned}
\phi_{i_{0}+1}^{(i)}(x)= & g_{i_{0}+1}^{(i)}\left\{|X| x \phi_{i_{0}}^{(i)}(x)+\beta_{i_{0}}^{(i)}\left(m_{0}+\cdots+m_{\overline{n_{i+1}}}\right) x\right. \\
& \left.-f_{i_{0}-1}^{(i)} \phi_{i_{0}-1}^{(i)}(x)-e_{i_{0}}^{(i)} \phi_{i_{0}}^{(i)}(x)\right\},
\end{aligned}
$$




$$
\beta_{i_{0}+1}^{(i)}=-g_{i_{0}+1}^{(i)^{-1}}\left(f_{i_{0}-1}^{(i)} \beta_{i_{0}-1}^{(i)}+e_{i_{0}}^{(i)} \beta_{i_{0}}^{(i)}\right) .
$$

Then

$$
E_{\overline{n_{i+1}}+i_{0}+1}=\phi_{i_{0}+1}^{(i)}\left(E_{\overline{n_{i+1}}+1}\right)+\beta_{i_{0}+1}^{(i)}\left(E_{0}+\cdots+E_{\overline{n_{i+1}}}\right) .
$$

Note here that $\phi_{i_{0}+1}^{(i)}(0)=0$. This shows (40).

(b) $\Rightarrow$ (a) We have to show (43)-(46). By (41), (43), and (46) hold. Let $i=t$. Then we only need to show (45) for $1 \leq i_{0} \leq n_{t}$. By our assumption, $E_{i_{0}}=\phi_{i_{0}}^{(t)}\left(E_{1}\right)$ for $0 \leq i_{0} \leq n_{t}$. Since $x \phi_{i_{0}}^{(t)}(x)$ is a linear combination of $\phi_{i_{0}+1}^{(t)}(x), \phi_{i_{0}}^{(t)}(x), \ldots, \phi_{0}^{(t)}(x), E_{1} \circ E_{i_{0}}$ is a linear combination of $E_{i_{0}+1}, E_{i_{0}}, \ldots, E_{0}$. Clearly, the coefficient of $E_{i_{0}+1}$ is not zero. This says $q_{1 i_{0}}^{j}=0$ for $j \geq i_{0}+2$, and $q_{1 i_{0}}^{j} \neq 0$ for $j=i_{0}+1$. As $m_{j} q_{k i}^{j}=m_{i} q_{k j}^{i}, q_{1 j}^{i}=$ $0 \Leftrightarrow q_{1 i}^{j}=0$. So $q_{1 i_{0}}^{j}=0$ if $\left|j-i_{0}\right| \geq 2$, and $q_{1 i_{0}}^{j} \neq 0$ if $\left|j-i_{0}\right|=1$.

Let $i \leq t-1$. First, we show (45). By our assumption (40) and (41),

$$
\begin{aligned}
& E_{\overline{n_{i+1}}+1} \circ E_{\overline{n_{i+1}}+i_{0}} \\
= & \phi_{i_{0}}^{(i)}\left(E_{\overline{n_{i+1}}+1}\right) \circ E_{\overline{n_{i+1}}+1}+|X|^{-1} \beta_{i_{0}}^{(i)}\left(m_{0}+\cdots+m_{\overline{n_{i+1}}}\right) E_{\overline{n_{i+1}}+1} .
\end{aligned}
$$

So, if we put

$$
x \phi_{i_{0}}^{(i)}(x)+|X|^{-1} \beta_{i_{0}}^{(i)}\left(m_{0}+\cdots+m_{\overline{n_{i+1}}}\right) x=\sum_{j=0}^{i_{0}+1} \varepsilon_{j} \phi_{j}^{(i)}(x)
$$

for some $\varepsilon_{j}$ with $\varepsilon_{i_{0}+1} \neq 0$, then, by $(40)$,

$$
\begin{aligned}
& E_{\overline{n_{i+1}}+1} \circ E_{\overline{n_{i+1}}+i_{0}} \\
= & -\sum_{j=0}^{i_{0}+1} \varepsilon_{j} \beta_{j}^{(i)}\left(E_{0}+\cdots+E_{\overline{n_{i+1}}}\right)+\sum_{j=1}^{i_{0}+1} \varepsilon_{j} E_{\overline{n_{i+1}}+j} .
\end{aligned}
$$

By the same argument as above, $q_{\overline{n_{i+1}}+1, \overline{n_{i+1}}+i_{0}}^{\overline{n_{i+1}}+j_{0}}=0$ if $\left|j_{0}-i_{0}\right| \geq 2$, and $q_{\overline{n_{i+1}}+1, \overline{n_{i+1}}+i_{0}}^{\overline{n_{i+1}}+j_{0}} \neq 0$ if $\left|j_{0}-i_{0}\right|=1$. Next, we show (44). By (40) with $i_{0}=2$, we have:

$$
E_{\overline{n_{i+1}}+2}=|X| e E_{\overline{n_{i+1}}+1} \circ E_{\overline{n_{i+1}}+1}+f E_{\overline{n_{i+1}}+1}+\beta_{2}^{(i)}\left(E_{0}+\cdots+E_{\overline{n_{i+1}}}\right)
$$

for some $e, f$ with $e \neq 0$. So, taking the proof of Theorem 7 into account, we get:

$E_{\overline{n_{i+1}}+1} \circ E_{\overline{n_{i+1}}+1}=|X|^{-1}\left\{m^{(i)}\left(E_{0}+\cdots+E_{\overline{n_{i+1}}}\right)+e_{1}^{(i)} E_{\overline{n_{i+1}}+1}+g_{2}^{(i)} E_{\overline{n_{i+1}}+2}\right\}$

with $g_{2}^{(i)} \neq 0$, where we put $e_{1}^{(i)}=-e^{-1} f, g_{2}^{(i)}=e^{-1}$.

(b) $\Leftrightarrow$ (c) This is straightforward.

(b) $\Leftrightarrow$ (d) For $i=1, \ldots, t, 1 \leq i_{0} \leq n_{i}$, or $i=t, i_{0}=0$, put

$$
\Phi_{i_{0}}^{(i)}(x)=\left|X_{1}\right| \cdots\left|X_{i-1}\right| \phi_{i_{0}}^{(i)}\left(\left(\left|X_{1}\right| \cdots\left|X_{i-1}\right|\right)^{-1} x\right)+\beta_{i_{0}}^{(i)}\left|X_{i}\right|^{-1} .
$$

Then we see that (40) is equivalent to:

$$
E_{0}^{(1)} \otimes \cdots \otimes E_{0}^{(i-1)} \otimes \Phi_{i_{0}}^{(i)}\left(E_{1}^{(i)}\right) \otimes I \otimes \cdots \otimes I
$$




$$
=E_{0}^{(i)} \otimes \cdots \otimes E_{0}^{(i-1)} \otimes E_{i_{0}}^{(i)} \otimes I \otimes \cdots \otimes I .
$$

In view of Lemma 12 , this in turn is equivalent to $E_{i_{0}}^{(i)}=\Phi_{i_{0}}^{(i)}\left(E_{1}^{(i)}\right)$ (cf. $\quad[2$, pp. 193-194]).

\section{An example}

Let $\mathfrak{X}=\left(X,\left\{R_{j}\right\}_{j=0}^{n}\right)$ be any symmetric association scheme. Let $\mathcal{A}$ be the Bose-Mesner algebra of $\mathfrak{X}$ generated by the adjacency matrices $A_{0}, A_{1}, \ldots, A_{n}$ $\in M_{|X|}(\mathbb{C}), p_{j l}^{k}$ the intersection numbers of $\mathfrak{X}, L_{j}=\left(p_{j l}^{k}\right)(j=0,1, \ldots, n)$ the intersection matrices of $\mathfrak{X}$, and let $\mathcal{B}$ be the subalgebra of $M_{n+1}(\mathbb{C})$ generated by $L_{0}, L_{1}, \ldots, L_{n}$. Then the map $\mathcal{A} \rightarrow \mathcal{B}\left(A_{i} \mapsto L_{i}\right)$ is an isomorphism as algebras over $\mathbb{C}$.

In view of this isomorphism and since $|X|$ is much bigger then $n+1$, we will express the modified polynomial relations for $H(4,3,5 ; 2)$ below in terms of intersection matrices. For this example, observe that $|X|=4096$, while $n+1=13$.

Now, we consider the weak Hamming scheme $H(4,3,5 ; 2)=H(4,2) \imath H(3,2)$ 乙 $H(5,2)$, which is associated with the poset $\mathbb{P}_{0}=4 \mathbf{1} \oplus 3 \mathbf{1} \oplus 5 \mathbf{1}$.

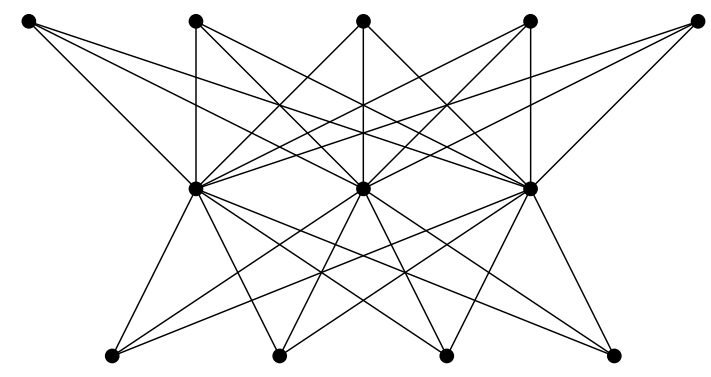

The $P$-matrices of $H(4,2), H(3,2), H(5,2)$ are respectively given by $\left(\begin{array}{ccccc}1 & 4 & 6 & 4 & 1 \\ 1 & 2 & 0 & -2 & -1 \\ 1 & 0 & -2 & 0 & 1 \\ 1 & -2 & 0 & 2 & -1 \\ 1 & -4 & 6 & -4 & 1\end{array}\right),\left(\begin{array}{cccc}1 & 3 & 3 & 1 \\ 1 & 1 & -1 & -1 \\ 1 & -1 & -1 & 1 \\ 1 & -3 & 3 & -1\end{array}\right),\left(\begin{array}{cccccc}1 & 5 & 10 & 10 & 5 & 1 \\ 1 & 3 & 2 & -2 & -3 & -1 \\ 1 & 1 & -2 & 2 & 1 & 1 \\ 1 & -1 & -2 & 2 & 1 & -1 \\ 1 & -3 & 2 & 2 & -3 & 1 \\ 1 & -5 & 10 & -10 & 5 & -1\end{array}\right)$, and the $P$-matrix of $H(4,3,5 ; 2)$ is

$$
\left(\begin{array}{ccccccccccccc}
1 & 4 & 6 & 4 & 1 & 48 & 48 & 16 & 640 & 1280 & 1280 & 640 & 128 \\
1 & 4 & 6 & 4 & 1 & 48 & 48 & 16 & 384 & 256 & -256 & -384 & -128 \\
1 & 4 & 6 & 4 & 1 & 48 & 48 & 16 & 128 & -256 & -256 & 128 & 128 \\
1 & 4 & 6 & 4 & 1 & 48 & 48 & 16 & -128 & -256 & 256 & 128 & -128 \\
1 & 4 & 6 & 4 & 1 & 48 & 48 & 16 & -384 & 256 & 256 & -384 & 128 \\
1 & 4 & 6 & 4 & 1 & 48 & 48 & 16 & -640 & 1280 & -1280 & 640 & -128 \\
1 & 4 & 6 & 4 & 1 & 16 & -16 & -16 & 0 & 0 & 0 & 0 & 0 \\
1 & 4 & 6 & 4 & 1 & -16 & -16 & 16 & 0 & 0 & 0 & 0 & 0 \\
1 & 4 & 6 & 4 & 1 & -48 & 48 & -16 & 0 & 0 & 0 & 0 & 0 \\
1 & 2 & 0 & -2 & -1 & 0 & 0 & 0 & 0 & 0 & 0 & 0 & 0 \\
1 & 0 & -2 & 0 & 1 & 0 & 0 & 0 & 0 & 0 & 0 & 0 & 0 \\
1 & -2 & 0 & 2 & -1 & 0 & 0 & 0 & 0 & 0 & 0 & 0 & 0 \\
1 & -4 & 6 & -4 & 1 & 0 & 0 & 0 & 0 & 0 & 0 & 0 & 0
\end{array}\right) .
$$


Intersection matrices $L_{i}(i=0,1, \ldots, 12)$ are respectively given by:

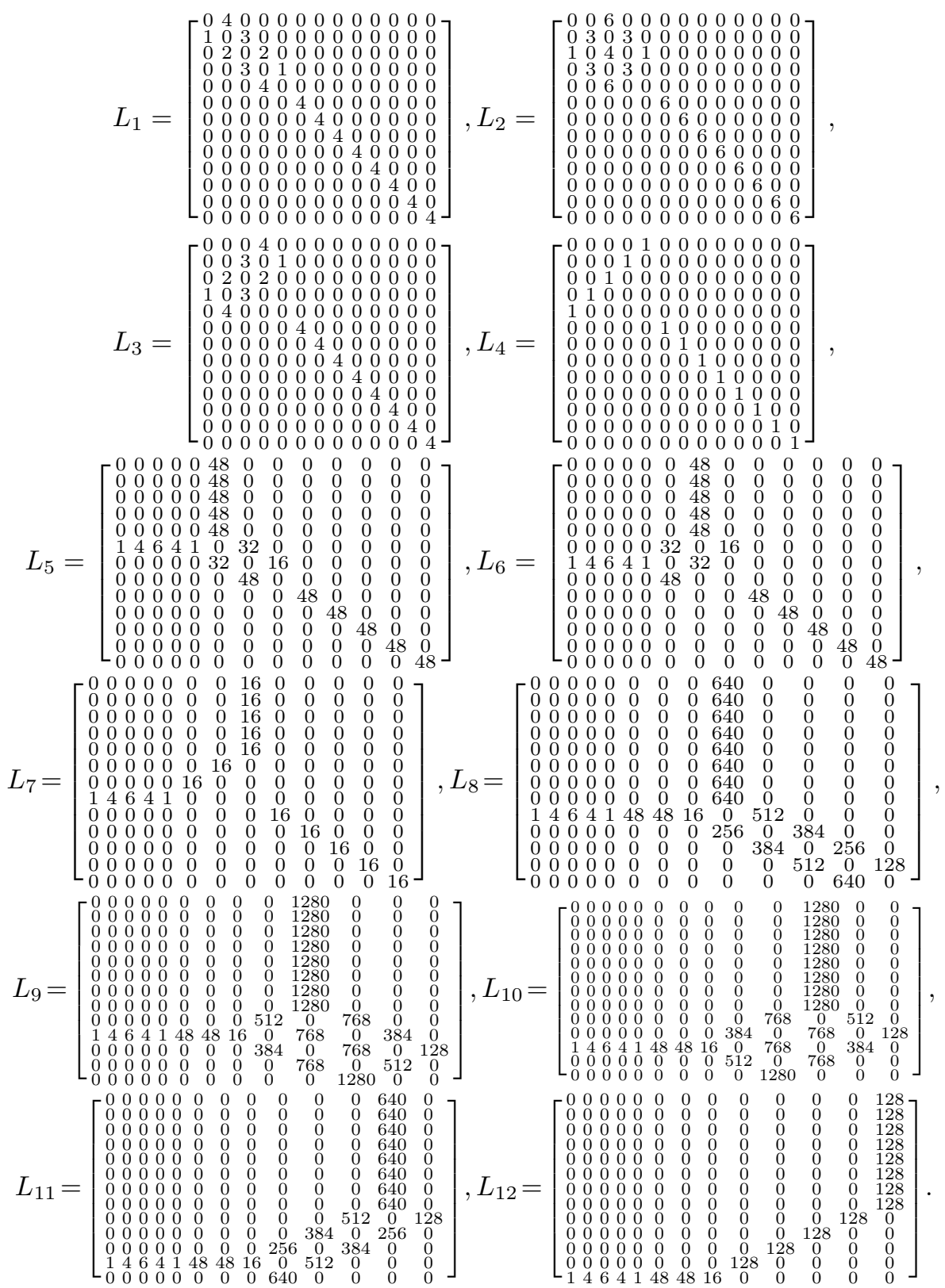

Finally, we have the result about modified polynomial relations of intersection matrices (cf. Theorem 2(c)).

$$
L_{1}, L_{2}=\frac{1}{2} L_{1}^{2}-2 I, L_{3}=\frac{1}{6} L_{1}^{3}-\frac{5}{3} L_{1}, L_{4}=\frac{1}{24} L_{1}^{4}-\frac{2}{3} L_{1}^{2}+I,
$$




$$
\begin{aligned}
& L_{5}, L_{6}=\frac{1}{32} L_{5}^{2}-\frac{3}{2}\left(I+L_{1}+\cdots+L_{4}\right), L_{7}=\frac{1}{1536} L_{5}^{3}-\frac{7}{6} L_{5}, \\
& L_{8}, L_{9}=\frac{1}{256} L_{8}^{2}-\frac{5}{2}\left(I+L_{1}+\cdots+L_{7}\right), L_{10}=\frac{1}{98304} L_{8}^{3}-\frac{13}{6} L_{8}, \\
& L_{11}=\frac{1}{50331648} L_{8}^{4}-\frac{11}{1536} L_{8}^{2}+\frac{15}{8}\left(I+L_{1}+\cdots+L_{7}\right), \\
& L_{12}=\frac{1}{32212254720} L_{8}^{5}-\frac{1}{65536} L_{8}^{3}+\frac{149}{120} L_{8} .
\end{aligned}
$$

Acknowledgment. We would like to thank Prof. J. Koolen and Dr. H. Tanaka for their help during the preparation of this paper.

\section{References}

[1] S. Bang and S. Y. Song, On generalized semidirect product of association schemes, Discrete Math. 303 (2005), no. 1-3, 5-16.

[2] E. Bannai and T. Ito, Algebraic combinatorics. I, Association schemes. The Benjamin/Cummings Publishing Co., Inc., Menlo Park, CA, 1984.

[3] A. E. Brouwer, A. M. Cohen, and A. Neumaier, Distance-Regular Graphs, Ergebnisse der Mathematik und ihrer Grenzgebiete (3) [Results in Mathematics and Related Areas (3)], 18. Springer-Verlag, Berlin, 1989.

[4] R. A. Brualdi, J. Graves, and K. M. Lawrence, Codes with a poset metric, Discrete Math. 147 (1995), no. 1-3, 57-72.

[5] D. S. Kim, MacWilliams-type identities for fragment and sphere enumerators, European J. Combin. 28 (2007), no. 1, 273-302.

[6] _ Dual MacWilliams pair, IEEE Trans. Inform. Theory 51 (2005), no. 8, 29012905.

[7] H. K. Kim and D. Y. Oh, A classification of posets admitting the MacWilliams identity, IEEE Trans. Inform. Theory 51 (2005), no. 4, 1424-1431.

[8] _ Association schemes for poset metrics and MacWilliams duality, to submit.

[9] S. Y. Song, Fusion relation in products of association schemes, Graphs Combin. 18 (2002), no. 3, 655-665.

DAE SAN KIM

Department of Mathematics

SOGANG UNIVERSITY

SeOul 121-742, KoreA

E-mail address: dskim@sogang.ac.kr

Gil Chun Kim

Department of Mathematics

SOGANG UNIVERSITY

SeOul 121-742, KoreA

E-mail address: higen@sogang.ac.kr 